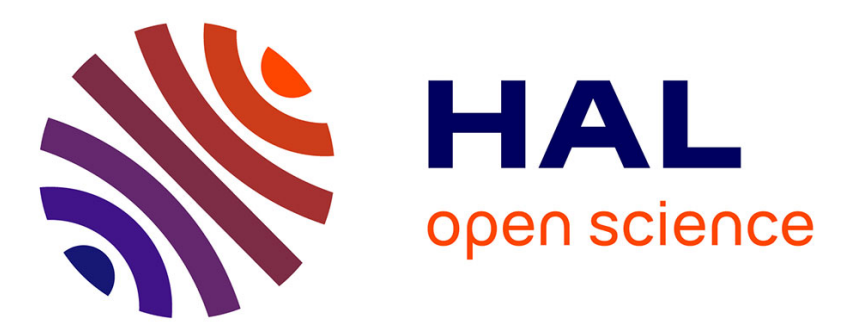

\title{
Phylogéographie de Neritina stumpffi Boettger, 1890 et Neritina canalis Sowerby, 1825 (Gastropoda, Cycloneritida, Neritidae)
}

\author{
Ahmed Abdou, Clara Lord, Philippe Keith, René Galzin
}

\section{To cite this version:}

Ahmed Abdou, Clara Lord, Philippe Keith, René Galzin. Phylogéographie de Neritina stumpffi Boettger, 1890 et Neritina canalis Sowerby, 1825 (Gastropoda, Cycloneritida, Neritidae). Zoosystema, 2019, 41 (12), pp.237-248. 10.5252/zoosystema2019v41a12 . hal-02549114

\author{
HAL Id: hal-02549114 \\ https://hal.science/hal-02549114
}

Submitted on 27 Apr 2020

HAL is a multi-disciplinary open access archive for the deposit and dissemination of scientific research documents, whether they are published or not. The documents may come from teaching and research institutions in France or abroad, or from public or private research centers.
L'archive ouverte pluridisciplinaire HAL, est destinée au dépôt et à la diffusion de documents scientifiques de niveau recherche, publiés ou non, émanant des établissements d'enseignement et de recherche français ou étrangers, des laboratoires publics ou privés. 

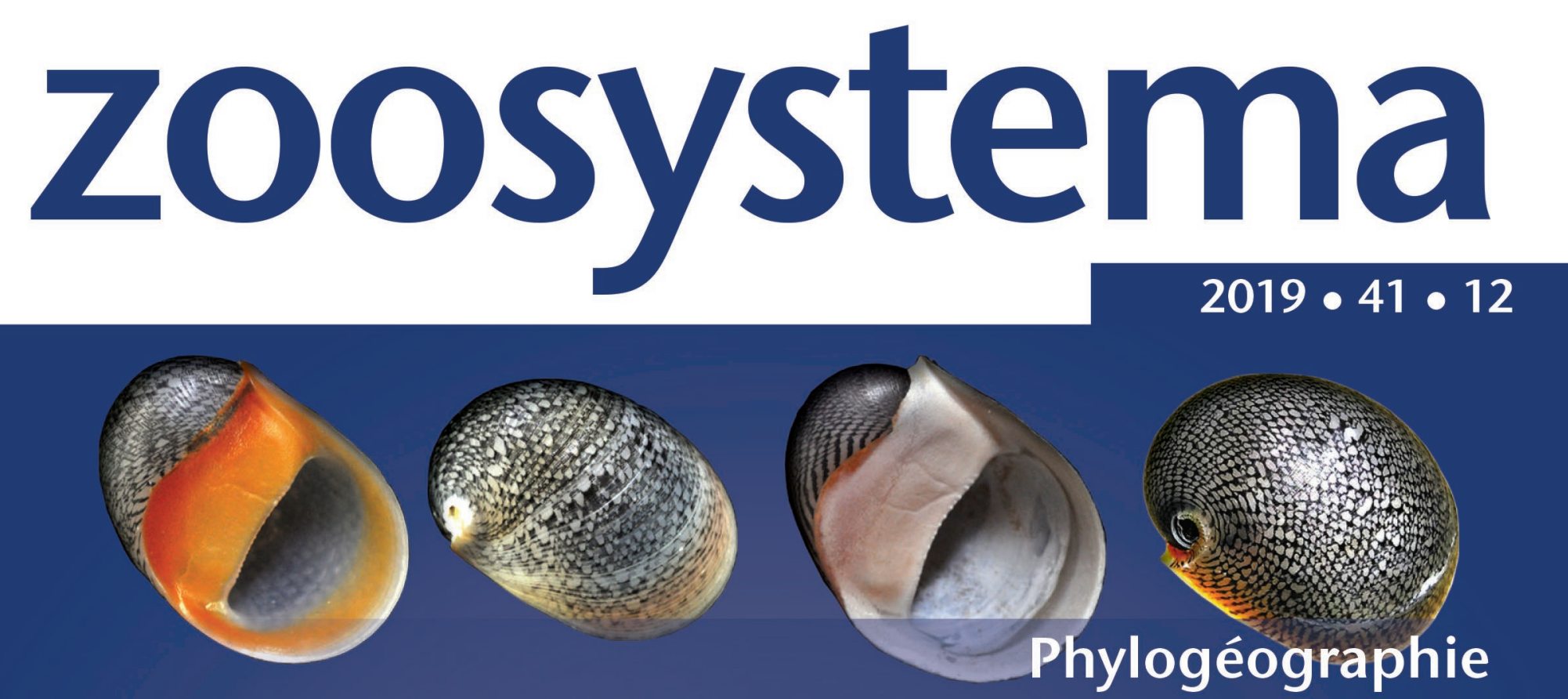
de Neritina stumpffi Boettger, 1890

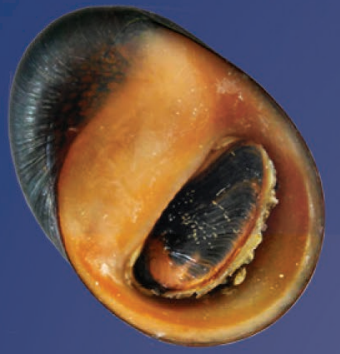
et Neritina canalis Sowerby, 1825 (Gastropoda, Cycloneritida, Neritidae)
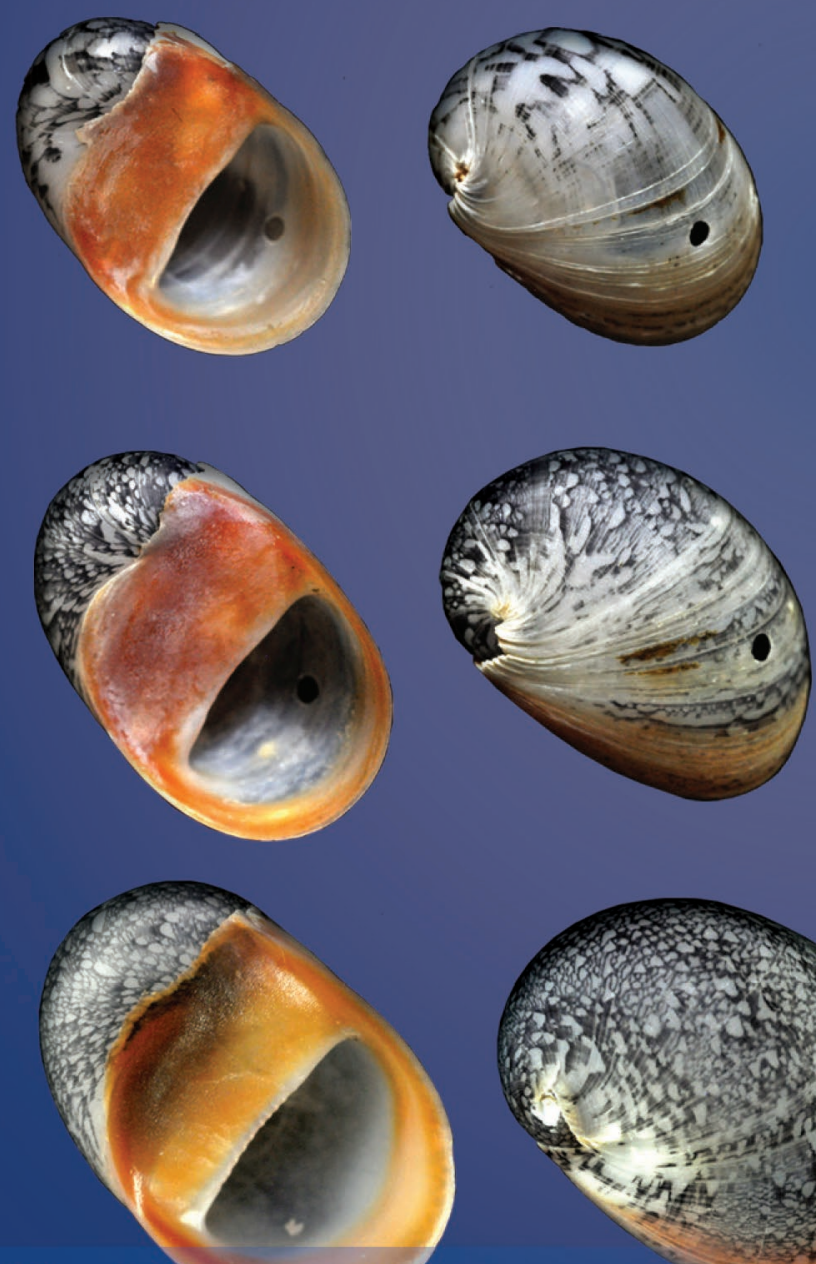

art. 41 (12) - Publié le 4 juin 2019 www.zoosystema.com
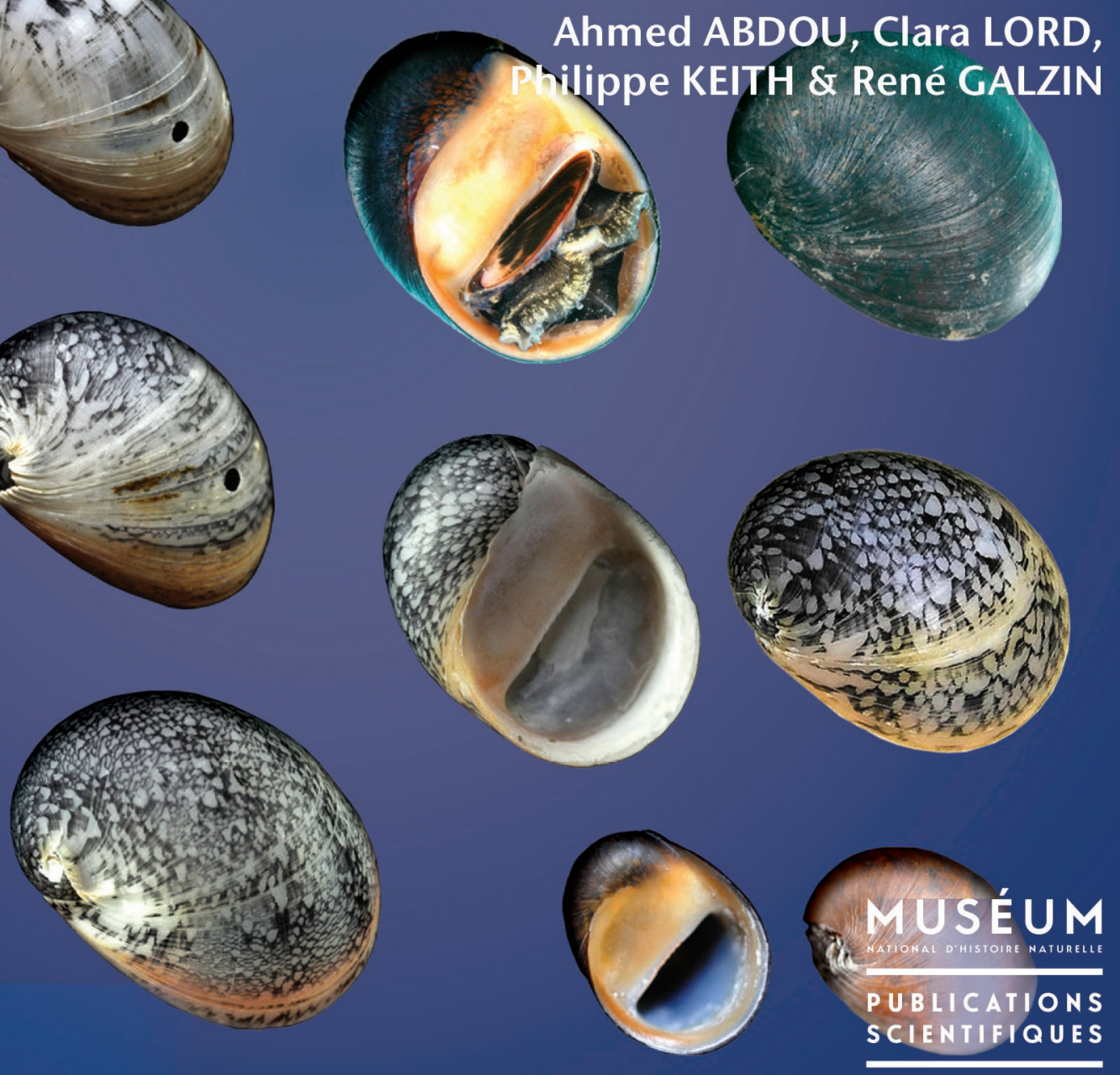
DiRECTEUR DE LA PUBlication: Bruno David

Président du Muséum national d'Histoire naturelle

RÉDACTRICE EN CHEF / EDITOR-IN-CHIEF: Laure Desutter-Grandcolas

AssistanTS DE RÉDACTION / AsSISTANT EDITORS: Anne Mabille (zoosyst@mnhn.fr), Emmanuel Côtez

Mise en page / Page Layout: Anne Mabille

COMITÉ SCIENTIFIQUE / SCIENTIFIC BOARD:

James Carpenter (AMNH, New York, États-Unis)

Maria Marta Cigliano (Museo de La Plata, La Plata, Argentine)

Henrik Enghoff (NHMD, Copenhague, Danemark)

Rafael Marquez (CSIC, Madrid, Espagne)

Peter Ng (University of Singapore)

Norman I. Platnick (AMNH, New York, États-Unis)

Jean-Yves Rasplus (INRA, Montferrier-sur-Lez, France)

Jean-François Silvain (IRD, Gif-sur-Yvette, France)

Wanda M. Weiner (Polish Academy of Sciences, Cracovie, Pologne)

John Wenzel (The Ohio State University, Columbus, États-Unis)

COUVERTURE / COVER:

Spécimens de Neritina canalis Sowerby, 1825 et Neritina stumpffi Boettger, 1890.

Zoosystema est indexé dans / Zoosystema is indexed in:

- Science Citation Index Expanded (SciSearch $\left.{ }^{\circledR}\right)$

- ISI Alerting Services ${ }^{\circledR}$

- Current Contents ${ }^{\circledR}$ / Agriculture, Biology, and Environmental Sciences ${ }^{\circledR}$

- Scopus ${ }^{\circledR}$

Zoosystema est distribué en version électronique par / Zoosystema is distributed electronically by:

- BioOne ${ }^{\circledR}$ (http://www.bioone.org)

Les articles ainsi que les nouveautés nomenclaturales publiés dans Zoosystema sont référencés par / Articles and nomenclatural novelties published in Zoosystema are referenced by:

- ZooBank ${ }^{\circledR}$ (http://zoobank.org)

Zoosystema est une revue en flux continu publiée par les Publications scientifiques du Muséum, Paris / Zoosystema is a fast track journal published by the Museum Science Press, Paris

Les Publications scientifiques du Muséum publient aussi / The Museum Science Press also publish:

Adansonia, Geodiversitas, Anthropozoologica, European Journal of Taxonomy, Naturae, Cryptogamie sous-sections Algologie, Bryologie, Mycologie.

Diffusion - Publications scientifiques Muséum national d'Histoire naturelle

CP 41 - 57 rue Cuvier F-75231 Paris cedex 05 (France)

Tél. : 33 (0)1 40794805 / Fax: 33 (0)1 40793840

diff.pub@mnhn.fr / http://sciencepress.mnhn.fr

(C) Publications scientifiques du Muséum national d'Histoire naturelle, Paris, 2019

ISSN (imprimé / print): 1280-9551/ ISSN (électronique / electronic): 1638-9387 


\title{
Phylogéographie de Neritina stumpffi Boettger, 1890 et Neritina canalis Sowerby, 1825 (Gastropoda, Cycloneritida, Neritidae)
}

\author{
Ahmed ABDOU \\ Clara LORD \\ Philippe KEITH \\ Unité Biologie des organismes et écosystèmes aquatiques (BOREA), \\ Muséum national d'Histoire naturelle, Sorbonne Université, \\ Université de Caen Normandie, Université des Antilles, CNRS, IRD, \\ case postale 26, 57 rue Cuvier, F-75231 Paris cedex 05 (France) \\ ahmed.abdou@mnhn.fr \\ René GALZIN \\ Laboratoire d'excellence Corail, USR 3278 CNRS-EPHE-UPVD, \\ Centre de Recherches insulaires et Observatoire de l'Environnement (CRIOBE), \\ BP 1013 Papetoai, 98729 Moorea, Polynésie française (France)
}

Soumis le 18 janvier 2018 | Accepté le 10 août 2018 | Publié le 4 juin 2019

MOTS CLÉS Phylogéographie, dispersion larvaire, océan Indien, océan Pacifique, gène COI.

KEY WORDS

Phylogeography, larval dispersion, Indian Ocean, Pacific Ocean, COI gene.
urn:Isid:zoobank.org:pub:578F05FA-D07C-43DD-9EAA-9391D7630C46

Abdou A., Lord C., Keith P. \& Galzin R. 2019. - Phylogéographie de Neritina stumpffi Boettger, 1890 et Neritina canalis Sowerby, 1825 (Gastropoda, Cycloneritida, Neritidae). Zoosystema 41 (12): 237-248. https://doi.org/10.5252/ zoosystema2019v41a12. http://zoosystema.com/41/12

\section{RÉSUMÉ}

La structuration génétique des populations de Neritina stumpffi Boettger, 1890 dans l'Indo-Pacifique et celle de Neritina canalis Sowerby, 1825 dans l'océan Pacifique, ont été étudiées par l'analyse de séquences partielles du gène mitochondrial COI. Le réseau d'haplotypes de $N$. stumpffi a montré une structuration génétique entre les deux océans, avec des haplotypes partagés. Concernant $N$. canalis, les populations du Pacifique ouest et du Pacifique central sont génétiquement structurées, sans haplotypes partagés. Le Triangle de corail semble avoir joué un rôle de barrière filtrante pour $N$. stumpffi, dont la présence dans les deux océans pourrait s'expliquer par la circulation du courant sud-équatorial. Une barrière à la dispersion de $N$. canalis pourrait également exister entre le Pacifique ouest et le Pacifique central.

\section{ABSTRACT}

Phylogeography of Neritina stumpffi Boettger, 1890 and Neritina canalis Sowerby, 1825 (Gastropoda, Cycloneritida, Neritidae).

The population genetic structure of Neritina stumpffi Boettger, 1890 in the Indo-Pacific and Neritina canalis Sowerby, 1825 in the Pacific Ocean were investigated using a fragment of the mitochondrial COI gene. The haplotype network of $N$. stumpffi showed a genetic structuration between the two oceans, with shared haplotypes. Concerning $N$. canalis, western Pacific and central Pacific populations are genetically structured, without shared haplotypes. The Coral Triangle appears to have played a filtering barrier role for $N$. stumpffi, whose presence in both oceans could be explained by the flow of the south-equatorial current. A barrier to the dispersal of $N$. canalis between the western and the central Pacific may also exist. 


\section{INTRODUCTION}

L'isolement géographique des îles océaniques joue en faveur de la structuration génétique des populations de certains groupes biologiques (Covich 2006; Crandall et al. 2010; Gaither et al. 2010). Au niveau de la faune terrestre, le cas d'un groupe à mobilité réduite comme les mollusques est assez démonstratif. Dans l'océan Indien comme dans l'océan Pacifique, plusieurs îles et archipels hébergent beaucoup d'espèces, voire de genres, endémiques. Sont endémiques aux Comores, par exemple, le genre Pseudelma Kobelt, 1904 (Streptaxidae Gray, 1860), composé de sept espèces (Abdou et al. 2008), ainsi que 11 espèces de Charopidae Hutton, 1884 (Muratov et al. 2005). Il y aurait $65 \%$ de mollusques terrestres endémiques à l'île Maurice (Griffiths \& Florens 2006) et la même proportion est observée aux Seychelles (Gerlach 2006). Dans le Pacifique, Abdou \& Bouchet (2000) rapportent que la plus riche radiation d'Endodontidae Pilsbry, 1895 de toute la Polynésie est constituée de quatre genres, dont trois endémiques et de 30 espèces endémiques, dans l'archipel des Gambier. Cowie et al. (1995) ont recensé 763 espèces indigènes dans l'archipel hawaiien, quasiment toutes endémiques. Enfin, selon Gargominy \& Fontaine (2014), 508 espèces ou sous-espèces (91\%) de mollusques terrestres et dulçaquicoles de Polynésie française seraient endémiques de ce territoire.

La situation est différente pour les gastéropodes aquatiques, marins et d'eau douce, dont le potentiel dispersif dépend essentiellement du mode de développement. Le développement direct semble prépondérant dans les écosystèmes dulçaquicoles où il est associé à une réduction de la mortalité larvaire en aval de l'habitat des adultes (Holthuis 1995; Crandall et al. 2010) alors que le développement à larves planctoniques prédomine dans le milieu marin, surtout chez les invertébrés tropicaux d'eau peu profonde (Vance 1973). Dans le cas des îles volcaniques, en raison de l'isolement, les systèmes dulçaquicoles ne peuvent être colonisés que par des espèces diadromes (Feutry et al. 2012). Pour être capables de coloniser ces milieux insulaires d'eau douce, les espèces diadromes amphidromes, particulièrement, dont la reproduction s'effectue en eau douce et la phase larvaire se déroule en mer durant quelques mois, n'ont d'autre choix que de produire des larves planctoniques, capables de retarder leur métamorphose en mer (Crandall et al. 2010; Lord et al. 2010, 2012 ; Abdou et al. 2015) jusqu'à trouver des habitats plus ou moins lointains dans des rivières propices à leur installation. En effet, le cycle de vie amphidrome consiste en une migration des larves, juste après éclosion, vers l'océan où elles se développent durant un temps variable selon les espèces, avant de se regrouper au niveau des estuaires, de se métamorphoser en juvéniles, puis de migrer vers les habitats des adultes en amont (McDowall 1992; Myers et al. 2000; Kano \& Kase 2003, 2004; McDowall 2007; Kano 2009; McDowall 2010; Crandall et al. 2010; Abdou et al. 2015). Ce cycle de vie existe essentiellement dans les petites îles tropicales et sub-tropicales, souvent jeunes et volcaniques, dans lesquelles les habitats d'eau douce sont très instables, et soumis à de fortes perturbations climatiques (McDowall 2007, 2010).
Plusieurs études génétiques à grande échelle géographique ont montré que la persistance des populations diadromes dans les milieux insulaires d'eau douce dépend des événements de colonisation, qui sont tributaires, notamment, de la capacité de dispersion des espèces, des distances entre les habitats et des courants océaniques favorables (Crandall et al. 2010; Lord et al. 2012; Castelin et al. 2013; Feutry et al. 2013). La durée de vie larvaire (DVL) de ces espèces varie de quelques heures à une année environ pour les gastéropodes (Kano 2006; Kano \& Kase 2008 ; Castelin 2010 ; Crandall et al. 2010), et elle contribue à la dispersion sur des distances plus ou moins importantes.

Les espèces amphidromes, avec leur phase de dispersion larvaire marine, peuvent être plus largement distribuées, et donc la connectivité des populations plus grande, que de nombreuses espèces marines (Crandall et al. 2010 ; Page et al. 2013). D'après Page et al. (2013), la connectivité phylogéographique serait déterminée par une combinaison de facteurs intrinsèques biologiques (l'histoire de vie) et de facteurs physiques extrinsèques (courants/hydrographie) variables, qui garantissent le potentiel mais pas nécessairement le pouvoir de dispersion. Pour sa part, Hansen (1980) met plutôt en avant le rôle de la tolérance environnementale (espèces eurytopes vs sténotopes) comme facteur important pour la dispersion à différentes échelles spatiales. Certaines espèces ayant des exigences particulières en matière d'habitat, la spécificité de celui-ci et les exigences écologiques du stade adulte peuvent être déterminantes dans la répartition géographique des populations (Resh et al. 1990, 1992 ; Liu \& Resh 1997 ; Crandall et al. 2008 ; Ayre et al. 2009 ; White et al. 2010 ; Postaire et al. 2014; Abdou et al. 2015). Dans leur étude sur la colonisation et l'expansion démographique de la faune d'eau douce dans l'archipel hawaïen, Alda et al. (2016) suggèrent que c'est bien l'écologie (les exigences climatiques et/ou l'habitat) plutôt que la géologie (l'âge de l'île) qui régit les assemblages des communautés d'eau douce insulaires.

Dans un habitat donné, la connectivité entre les populations est donc régie notamment par les capacités de dispersion des organismes et la présence ou non de barrières biogéographiques (Gaither et al. 2010). La plus grande barrière indo-pacifique connue est l'archipel indo-australien (AIA) (Gaither et al. 2010 ; Lord et al. 2012; Castelin et al. 2013), qui a fortement impacté la distribution des populations marines lors des glaciations du Pléistocène. En raison des fluctuations du niveau de la mer, il a constitué une barrière physique intermittente ayant permis la formation de ponts reliant les systèmes fluviaux au Pléistocène (Voris 2000; de Bruyn et al. 2012; Fig. 1). Pendant cette période, les détroits de Torres, de la Sonde et de Malacca ont été fermés plusieurs fois (Voris 2000; Crandall et al. 2008; Taillebois et al. 2013), limitant ainsi la dispersion larvaire entre les deux océans (Fig. 1). D'après Rijsdijk et al. (2014), certaines îles ont vu leur taille réduite de plus de 50\% depuis la période du dernier maximum glaciaire, en raison de l'élévation du niveau de la mer. Cela a conduit à une perte importante de l'habitat côtier et une augmentation significative de l'isolement par l'augmentation des distances entre les îles. Cette barrière a donc joué un rôle de filtre aussi bien en son sein qu'entre le Pacifique et l'océan Indien. 


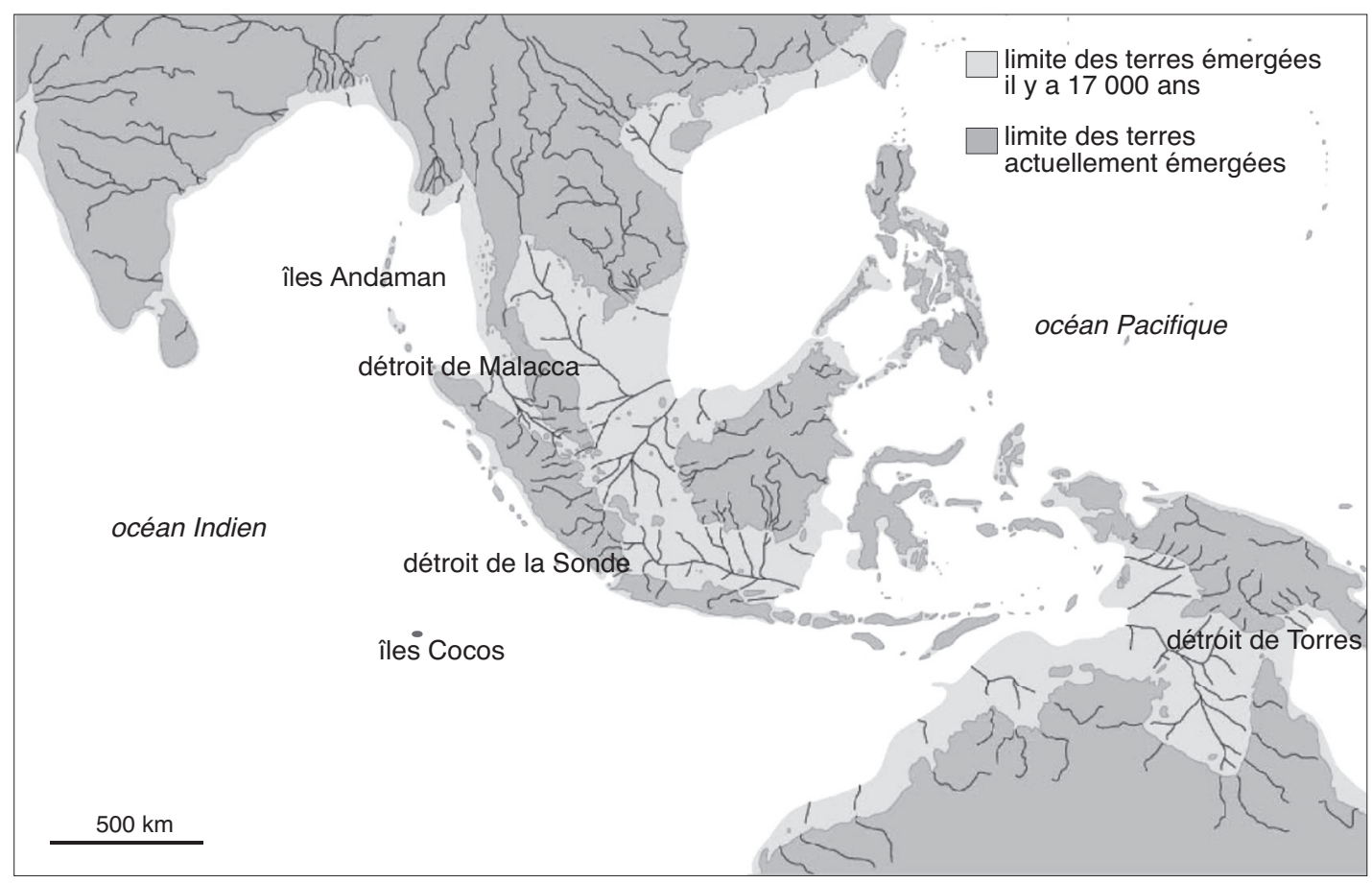

FIG. 1. - Carte de la région Indo-australienne au Pléistocène, lors du dernier maximum glaciaire, il y a 17000 ans environ. Le niveau de la mer était à 120 m en dessous du niveau actuel. Les détroits de Malacca, de la Sonde et de Torres, par exemple, étaient fermés. Ce niveau eustatique a varié de $120 \mathrm{~m}$ à $0 \mathrm{~m}$ durant le Pléistocène, avec toutes les profondeurs intermédiaires ayant chaque fois fermé ou ouvert un passage (rôle de barrière filtrante de l'AIA). Carte modifiée d'après Voris (2000), (c) 2000 Field Museum of Natural History, Chicago, Illinois, USA.

Compte tenu des capacités dispersives et des éventuels obstacles à la dispersion, la connectivité entre les populations d'une même espèce peut être indirectement évaluée par l'étude de la structure génétique entre ces populations. La distribution géographique des espèces est le reflet de cette connectivité. La phylogéographie permet donc de mettre en évidence l'existence ou non de barrières aux flux de gènes ou d'isolements géographiques (Avise et al. 1987; Avise 1998). Ces barrières peuvent être physiques, physiologiques, écologiques ou comportementales (Covich 2006). Elles peuvent être de plusieurs natures à la fois, comme c'est le cas, par exemple, pour Nerita atramentosa Reeve, 1855, une des rares espèces de Neritidae, autres que celles du genre Theodoxus Montfort, 1810, à s'être adaptée au climat tempéré (Ayre et al. 2009; Waters et al. 2005). Cette espèce montre une structure génétique est-ouest au sud de l'Australie, de part et d'autre du promontoire de Wilson, qui constitue une barrière physique paléogéographique. Sa distribution latitudinale au sud de l'Australie et dans l'île du nord de la Nouvelle-Zélande est, par contre, de nature écologique car liée à la température (Waters et al. 2005).

Neritina stumpffi Boettger, 1890 est une espèce de gastéropode Neritidae amphidrome qui était connue seulement de l'océan Indien occidental (Madagascar, Comores, Seychelles) jusqu'à ce que Abdou et al. (2017) découvrent sa présence dans le Pacifique (Japon, Guam, Nouvelle-Calédonie, Futuna). Neritina canalis Sowerby, 1825 a une large répartition dans le Pacifique ouest (PO) et central (PC), allant des Philippines aux Îles Marquises (Pointier \& Marquet 1990; Haynes 2001, 2005; Crandall et al. 2010). Ces deux espèces sont globalement semblables par la forme et les couleurs de leurs coquilles. Elles ont une coquille oblongue, de couleur marron à noir (Fig. 2). Neritina stumpffi se distingue de $N$. canalis par un opercule dont la face externe est brun foncé, presque noir (Boettger 1890 ; Starmühlner 1983), une cloison pariétale granuleuse, rouge à blanchâtre. L'opercule de certains échantillons présente, partant du noyau, une bande claire divisée en forme de Y. La seconde possède, quant à elle, une cloison pariétale jaune orange à rouge postérieurement et, généralement, un «canal » entre le bord pariétal et la terminaison supérieure du labre.

Bien qu'elles soient assez largement réparties, ces deux espèces ont été peu étudiées.

L'objectif de notre travail est d'étudier la structuration génétique des populations de Neritina stumpffi et de $N$. canalis au sein de leur aire de distribution i.e., respectivement l'IndoPacifique et l'océan Pacifique. Cette analyse permettra de visualiser l'existence ou non d'une rétention larvaire et donc la présence d'un flux de gènes à différentes échelles spatiales, entre les différentes localités. Ce travail sera plus particulièrement significatif pour la gestion et la conservation des espèces. En effet, selon que le flux des gènes soit transocéanique, régional ou très local, la gestion sera nécessairement différente.

\section{MATÉRIEL ET MÉTHODES}

\section{ÉCHANTILLONNAGE ET SÉQUENÇAGE}

Une bonne partie du matériel concernant l'océan Indien a été récoltée à l'occasion de différentes missions de terrain. 
C'est le cas des Comores, où deux campagnes ont eu lieu en 2005 et 2006. Des récoltes de Neritidae ont été réalisées du 30 octobre au 4 novembre 2005 à Mohéli (Mwali) et Anjouan (Ndzuwani) lors d'une mission d'inventaire financée par Conservation International (Keith et al. 2006). Une autre mission, conduite par l'Association réunionnaise de Développement de l'Aquaculture (ARDA) a eu lieu à Mayotte (Maore) du 13 au 21 mai 2006 (Valade et al. 2007). Des récoltes complémentaires ont été réalisées à Mohéli les 30 et 31 décembre 2013. Une mission spécifique organisée conjointement par l'UMR 7208 BOREA et l'USR 3278 CRIOBE nous a permis d'échantillonner à Mo'orea et Tahiti en Polynésie française durant le mois de février 2014. En outre, nous avons pu bénéficier d'échantillons collectés par des membres BOREA et par des partenaires de la zone indo-pacifique provenant des Seychelles, de Mayotte, de Nouvelle-Calédonie, de Futuna et du Japon (Okinawa) (Tableau 1; Fig. 3).

Sur le terrain, les nérites ont été essentiellement collectés à vue. Ils sont, en effet, pratiquement tous visibles à l'œil nu, vivants accrochés au substrat de blocs rocheux. Les spécimens ont été fixés à l'alcool à $70 \%$ ou à $95 \%$. Nous avons identifié les espèces, en nous aidant de la littérature, de la description d'origine (Boettger 1890) et des collections du Muséum national d'Histoire naturelle, Paris (MNHN).

En raison de la qualité de conservation de certains spécimens des Comores, l'analyse moléculaire n'a pu être réalisée que sur un nombre limité des échantillons issus des récoltes de 2005 et 2006. Un fragment de 609 pb du gène mitochondrial codant pour la première sous-unité de la Cytochrome oxydase I (COI) a été séquencé pour 58 spécimens, 34 de $N$. stumpffi et 24 de N. canalis (Tableau 1). Pour N. stumpffi (Fig. 2D-H), 28 échantillons sont issus de l'océan Indien (Comores, $\mathrm{N}=25$ et Seychelles, $\mathrm{N}=3$ ) et six de l'océan Pacifique (NouvelleCalédonie, $\mathrm{N}=2$; Japon, $\mathrm{N}=2$; Futuna, $\mathrm{N}=1$ et Guam, $\mathrm{N}=1$ ). Pour N. canalis (Fig. 2A-C), 16 échantillons sont de Mo'orea, un de Tahiti, cinq de Futuna, un du Vanuatu et un de Samoa. Cinq séquences ont été récupérées dans GenBank (AB477484, AB477485, AB477505 à AB477507), trois de N. stumpffi et deux de $N$. canalis (Kano 2009).

Pour chaque individu sélectionné, nous avons prélevé quelques milligrammes de tissu du muscle du pied. L'ADN génomique total a été extrait à l'aide du robot semi-automatique eppendorf "epMotion 5075" suivant le protocole du Kit NucleoSpin 96 Tissue de MACHEREY-NAGEL. L'ADN a été amplifié par PCR en utilisant les amorces universelles développées par Folmer et al. (1994), LCOI1490 (5'-GGTCAACAAATCATAAAGATATTGG-3') et HCO2198 (5'-TAAACTTCAGGGTGACCAAAAAATCA-3'). L'amplification a été réalisée dans un volume final d'environ $21 \mu$ l contenant $12,94 \mu \mathrm{l}$ d'eau stérile, $2 \mu \mathrm{l}$ de tampon TAQ, $1 \mu \mathrm{l}$ de DMSO (DiMéthyl SulfOxyde), $1 \mu \mathrm{l}$ de BSA (Sérum Albumine Bovine), 1,5 $\mu$ l de $\mathrm{MgCl}_{2}$, 0,80 $\mu$ lde Mix dNTP (DesoxyriboNucleotide TriPhosphate) à $6,6 \mathrm{mM}, 0,32 \mu \mathrm{l}$ de chaque amorce, $0,12 \mu \mathrm{l}$ de Taq polymérase (Qiagen), 1 à 1,5 $\mu \mathrm{l}$ d'ADN. La PCR a été réalisée à l'aide d'un thermocycleur Bio-Rad C1000 Touch ${ }^{\mathrm{TM}}$, selon le programme ci-après : trois minutes de dénaturation initiale à $95^{\circ} \mathrm{C}$, suivie de 40 cycles de dénaturation à $95^{\circ} \mathrm{C}$
TABLEAU 1. - Liste et localisation (archipels) des échantillons ayant fait l'objet de l'analyse moléculaire. Le numéro d'identification des specimens au Muséum national d'Histoire naturelle (MNHN) ainsi que celui des séquences dans GenBank sont indiqués.

\begin{tabular}{|c|c|c|c|c|}
\hline Localité & Année & $\mathrm{Nb}$ & MNHN & GenBank \\
\hline \multicolumn{5}{|c|}{ Neritina stumpffi Boettger, 1890} \\
\hline \multirow{3}{*}{$\begin{array}{l}\text { Anjouan, } \\
\text { (Comores) }\end{array}$} & \multirow[t]{3}{*}{2005} & & MNHN-IM-2013-62796 & MF407571 \\
\hline & & & MNHN-IM-2013-62797 & MF407648 \\
\hline & & & MNHN-IM-2013-62798 & MF407639 \\
\hline \multirow[t]{2}{*}{ Mohéli (Comores) } & \multirow[t]{2}{*}{2005} & & MNHN-IM-2013-62794 & MF407637 \\
\hline & & & MNHN-IM-2013-62795 & MF407638 \\
\hline \multirow[t]{2}{*}{ Mohéli (Comores) } & \multirow[t]{2}{*}{2008} & & MNHN-IM-2013-62792 & MF407619 \\
\hline & & & MNHN-IM-2013-62793 & MF407620 \\
\hline \multirow[t]{14}{*}{ Mohéli (Comores) } & \multirow[t]{14}{*}{2013} & 14 & MNHN-IM-2013-62778 & MF407618 \\
\hline & & & MNHN-IM-2013-62779 & MF407617 \\
\hline & & & MNHN-IM-2013-62780 & MF407636 \\
\hline & & & MNHN-IM-2013-62781 & MF407622 \\
\hline & & & MNHN-IM-2013-62782 & MF407624 \\
\hline & & & MNHN-IM-2013-62783 & MF407630 \\
\hline & & & MNHN-IM-2013-62784 & MF407631 \\
\hline & & & MNHN-IM-2013-62785 & MF407626 \\
\hline & & & MNHN-IM-2013-62786 & MF407615 \\
\hline & & & MNHN-IM-2013-62787 & MF407632 \\
\hline & & & MNHN-IM-2013-62788 & MF407627 \\
\hline & & & MNHN-IM-2013-62789 & MF407633 \\
\hline & & & MNHN-IM-2013-62790 & MF407628 \\
\hline & & & MNHN-IM-2013-62791 & MF407629 \\
\hline Mayotte (Comores) & 2006 & & MNHN-IM-2013-62802 & MF407642 \\
\hline \multirow[t]{3}{*}{ Mayotte (Comores) } & \multirow[t]{3}{*}{2014} & & MNHN-IM-2013-62799 & MF407616 \\
\hline & & & MNHN-IM-2013-62800 & MF407634 \\
\hline & & & MNHN-IM-2013-62801 & MF407635 \\
\hline \multirow[t]{3}{*}{ Seychelles } & \multirow[t]{3}{*}{2012} & & MNHN-IM-2013-62803 & MF407625 \\
\hline & & & MNHN-IM-2013-62804 & MF407640 \\
\hline & & & MNHN-IM-2013-62805 & MF407641 \\
\hline \multirow[t]{2}{*}{ Nouvelle-Calédonie } & \multirow[t]{2}{*}{2013} & & MNHN-IM-2013-62806 & MF407645 \\
\hline & & & MNHN-IM-2013-62807 & MF407623 \\
\hline & \multirow[t]{3}{*}{2014} & & MNHN-IM-2013-62809 & MF407621 \\
\hline \multirow[t]{2}{*}{ Okinawa (Japon) } & & 2 & - & AB477505 \\
\hline & & & & AB477506 \\
\hline Guam (Micronésie) & & 1 & - & AB477507 \\
\hline
\end{tabular}

Neritina canalis Sowerby, 1825

\begin{tabular}{lllll}
\hline Vanuatu & 2006 & 1 & - & AB477484
\end{tabular}

Samoa

5 MNHN-IM-2013-62820 MF407598 MNHN-IM-2013-62821 MF407599 MNHN-IM-2013-62822 MF407600 MNHN-IM-2013-62823 MF407601 MNHN-IM-2013-62824 MF407602 Tahiti (Polynésie) $2014 \quad 1$ MNHN-IM-2013-62825 MF407585 Mo'orea 2014 (Polynésie)

16 MNHN-IM-2013-62826 MF407587 MNHN-IM-2013-62827 MF407588 MNHN-IM-2013-62828 MF407589 MNHN-IM-2013-62829 MF407590 MNHN-IM-2013-62830 MF407591 MNHN-IM-2013-62831 MF407603 MNHN-IM-2013-62832 MF407586 MNHN-IM-2013-62833 MF407597 MNHN-IM-2013-62834 MF407596 MNHN-IM-2013-62835 MF407604 MNHN-IM-2013-62836 MF407583 MNHN-IM-2013-62837 MF407584 MNHN-IM-2013-62838 MF407592 MNHN-IM-2013-62839 MF407593 MNHN-IM-2013-62840 MF407594 MNHN-IM-2013-62841 MF407595 

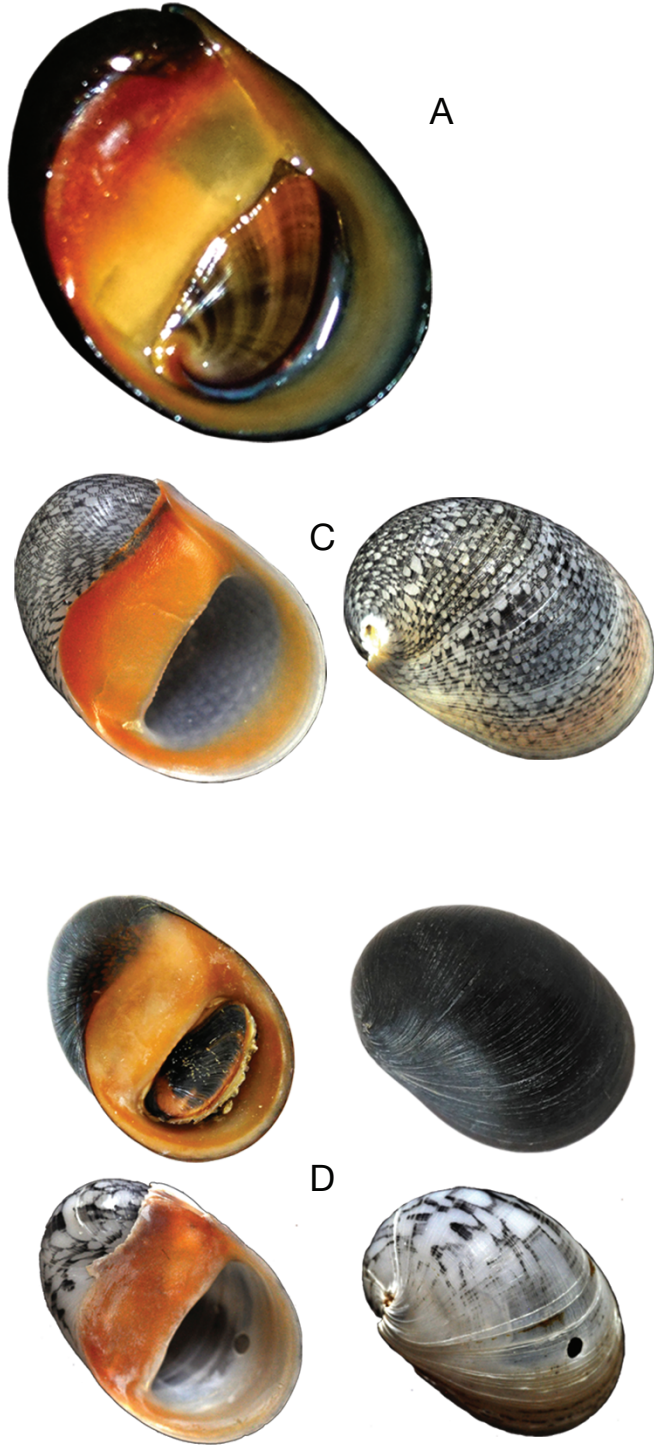

\section{D}
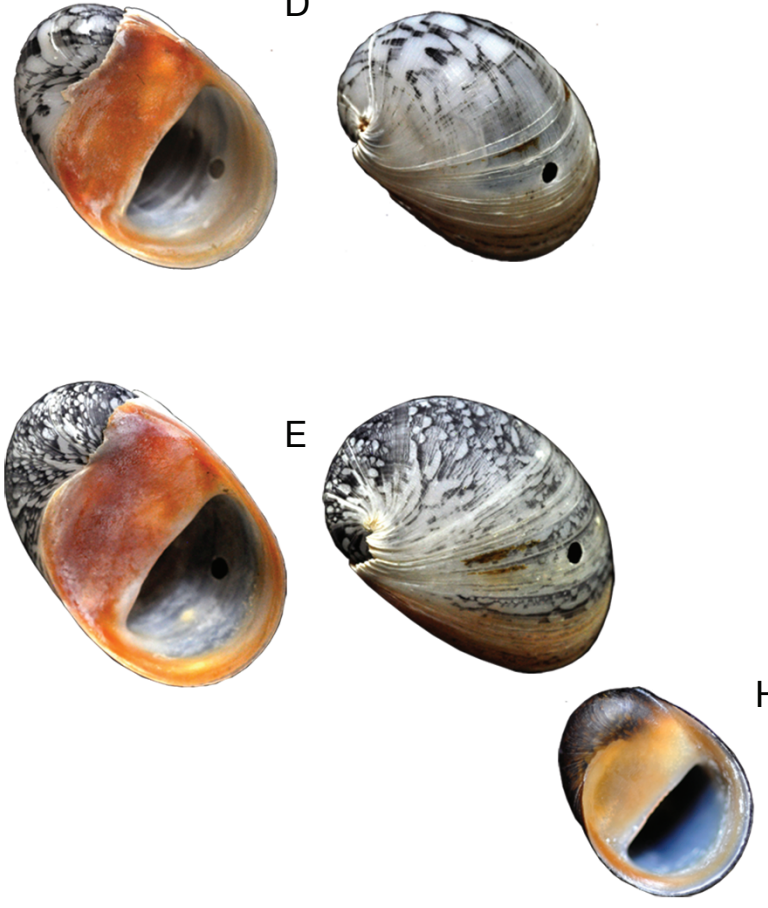
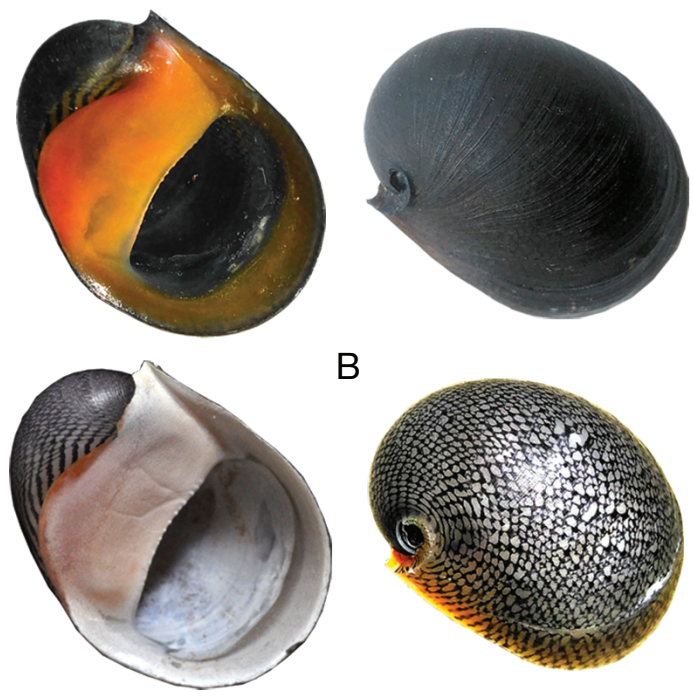

B
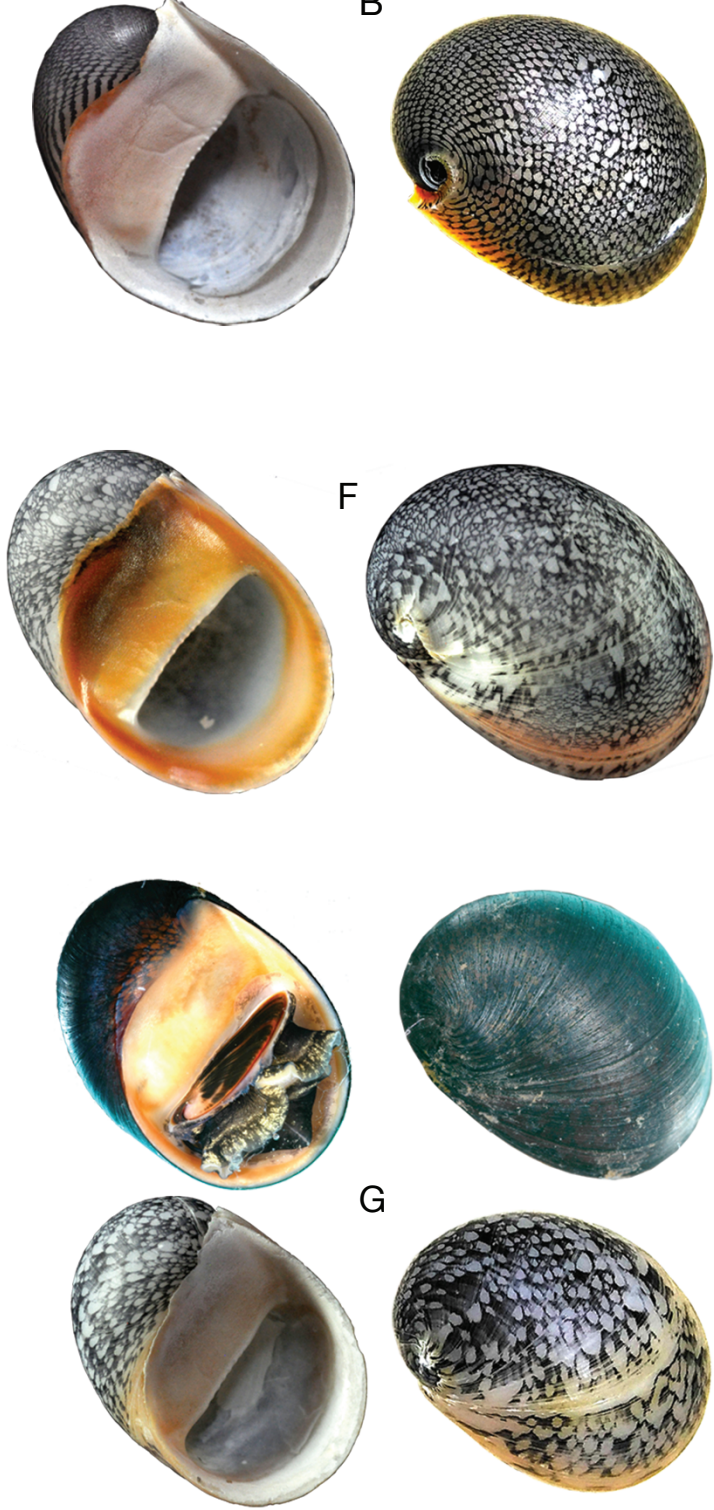

G

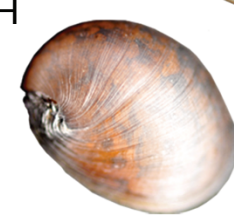

FIG. 2. - A-C, Neritina canalis Sowerby, 1825 : A, MNHN-IM-2013-62837, Mo'orea; B, MNHN-IM-2013-62829 Mo'orea; C, MNHN-IM-2013-62821, Futuna; D-H, N. stumpffi Boettger, 1890: D, MNHN-IM-2013-62806, Nouvelle-Calédonie; E, MNHN-IM-2013-62808, Nouvelle-Calédonie; F, MNHN-IM-2013-62809, Futuna; G, MNHN-IM-2013-62796, Comores; H, MNHN-IM-2013-62803, Seychelles. Les échantillons sont présentés avec le périostracum (A, H, et position haute de $\mathbf{B}, \mathbf{D}, \mathbf{G})$ ou sans le périostracum $(\mathbf{C}, \mathbf{E}, \mathbf{F}$, et position basse de $\mathbf{B}, \mathbf{D}, \mathbf{G})$ après un traitement à l'eau de javel, montrant ainsi les motifs réels de la coquille, produits au niveau de la couche calcitique externe. Hauteur des spécimens : A, 28,40 mm ; B, 21,10 mm; C, 19,86 mm ; D, 17,18 mm; E, $19,66 \mathrm{~mm}$; F, 22,11 mm; G, $20 \mathrm{~mm} ; \mathrm{H}, 14,78 \mathrm{~mm}$ (la hauteur des échantillons a été mesurée à l'aide d'un pied à coulisse électronique " Absolute digimatic caliper ", model n CD-15DC, de Mitutoyo corp, avec une marge d'erreur de 0,02 $\mathrm{mm}$ ). 


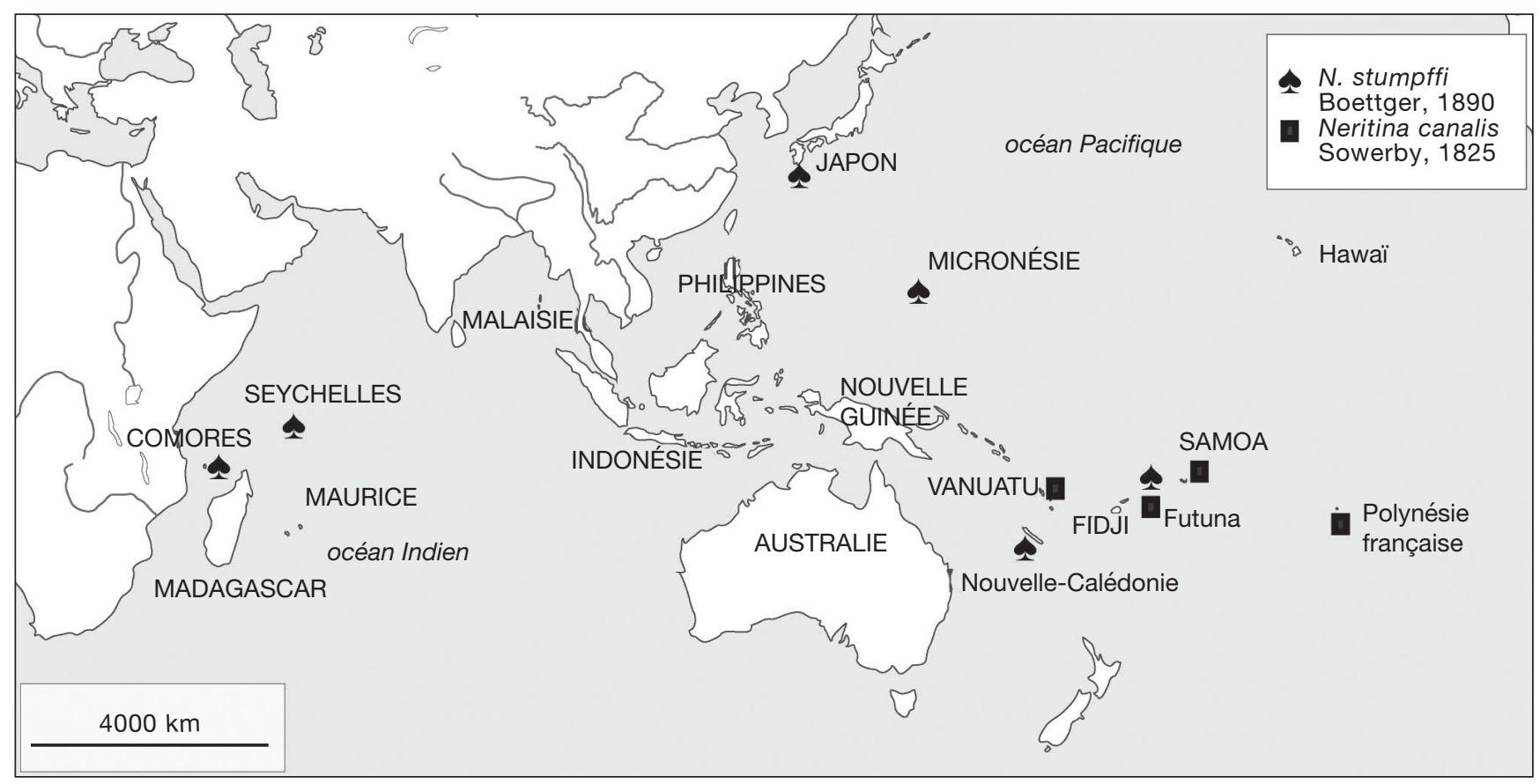

FIG. 3. - Localités de provenance des échantillons étudiés pour les deux espèces.

pour 30 secondes, hybridation $40-42^{\circ} \mathrm{C}$ pour 30 secondes, élongation à $72^{\circ} \mathrm{C}$ pour 45 secondes, suivis d'une élongation finale de 5 minutes à $72^{\circ} \mathrm{C}$. Les produits de PCR ont été visualisés par migration électrophorétique sur un gel d'agarose à 1,5\%, préparé avec du tampon TBE (Tris, Borate, EDTA) et un agent intercalant de l'ADN, le BET (Bromure d'Ethidium). Le séquençage des produits PCR a été réalisé par Eurofins (http://www.eurofins.fr) en utilisant les mêmes amorces.

Les séquences partielles du gène COI ont été vérifiées et nettoyées et les chromatogrammes des deux brins (forward et reverse) ont été comparés à l'aide du logiciel CodonCode Aligner 5.0.2 (Codon Code Corporation). Les séquences ont été alignées par ClustalW à l'aide du logiciel MEGA 6.0 (Tamura et al. 2013).

\section{Analyse des données}

Le petit nombre d'échantillons par île ne pouvant pas être utilisé pour les analyses de populations à l'échelle locale, nous avons fait le choix de répartir les îles en deux groupes distincts pour chaque espèce. Ces deux groupes matérialisent les deux populations observées. Il s'agit des groupes océan Indien (Comores, Seychelles) et océan Pacifique (Japon, Guam, Nouvelle-Calédonie, Futuna) pour N. stumpffi ; Pacifique ouest (Vanuatu, Samoa, Futuna) et Pacifique central (Polynésie française) pour $N$. canalis.

Les indices de diversité tels que le nombre d'haplotypes (nd), la diversité haplotypique (h), qui mesure l'unicité d'un haplotype particulier dans une population donnée et la diversité nucléotidique $(\pi)$, qui mesure le degré de polymorphisme au sein d'une population, ont été estimés avec Arlequin v3.5.2.2 (Excoffier \& Lischer 2010). La recherche d'haplotypes partagés par plusieurs individus a été effectuée à l'aide du logiciel
DnaSP v5.1 (Librado \& Rozas 2009). Pour visualiser les relations généalogiques entre les haplotypes et leur répartition géographique, des réseaux d'haplotypes ont été construits selon la méthode du Median-Joining implémentée dans Network v4.6.1.4 (Bandelt et al. 1999), en utilisant les paramètres par défaut. Pour évaluer le niveau de structure génétique entre les différents groupes de localités, l'indice de différenciation génétique $F_{S T}$ (Wright 1951, 1978) a été estimé, à partir d'une analyse de variance/covariance moléculaire (AMOVA : Analysis of MOlecular VAriance, Excoffier et al. 1992) implémentée dans le programme Arlequin v3.5.2.2 (Excoffier \& Lischer 2010). Le $F_{S T}$ est testé par rapport à une distribution théorique obtenue par 1000 jeux de données simulés par permutation des individus entre les groupes prédéfinis.

\section{RÉSULTATS}

Sur les 34 séquences de 609 pb du COI obtenues pour $N$. stumpffi, nous avons obtenu 25 haplotypes et 56 (9\%) sites polymorphes (nsp). La diversité haplotypique (h) est très élevée avec la valeur maximale $(1,0)$ dans l'océan Pacifique; la diversité nucléotidique $(\pi)$ est, en revanche, faible mais relativement plus élevée dans l'océan Indien (voir Tableau 2).

Le réseau d'haplotypes (Fig. 4) montre deux entités génétiques fortement structurées $\left(\mathrm{F}_{\mathrm{ST}}=0,288 ; \mathrm{p}=0,014\right)$, mais non superposables à la répartition géographique.w Le premier haplogroupe est composé des haplotypes issus du Pacifique (Japon, Guam, Nouvelle-Calédonie, Futuna) et d'haplotypes issus de l'océan Indien (11 des Comores et un des Seychelles). Le second haplogroupe est uniquement composé d'haplotypes provenant de l'océan Indien. Un des haplotypes du second 


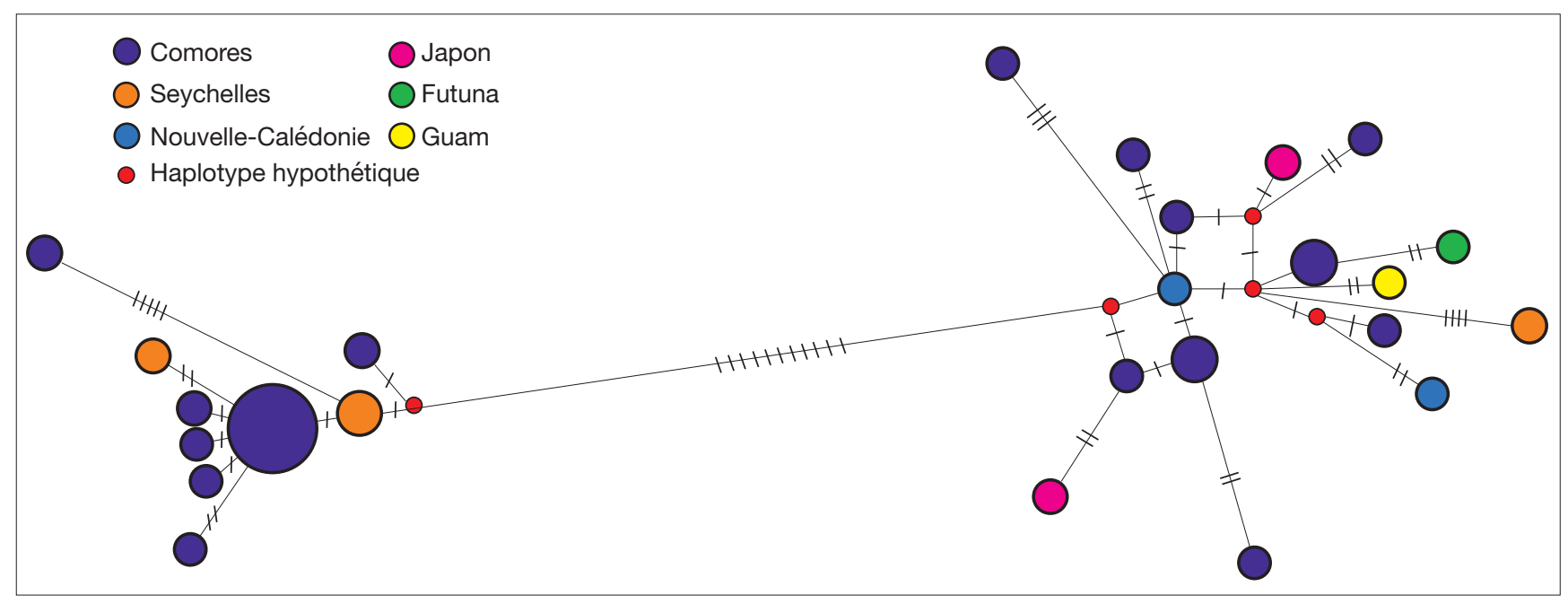

FIG. 4. - Réseau d'haplotypes de Neritina stumpffi Boettger, 1890 selon la méthode du median-joining. Les cercles sont proportionnels à la fréquence des occurences, le nombre de mutations est indiqué sur les branches par des tirets.

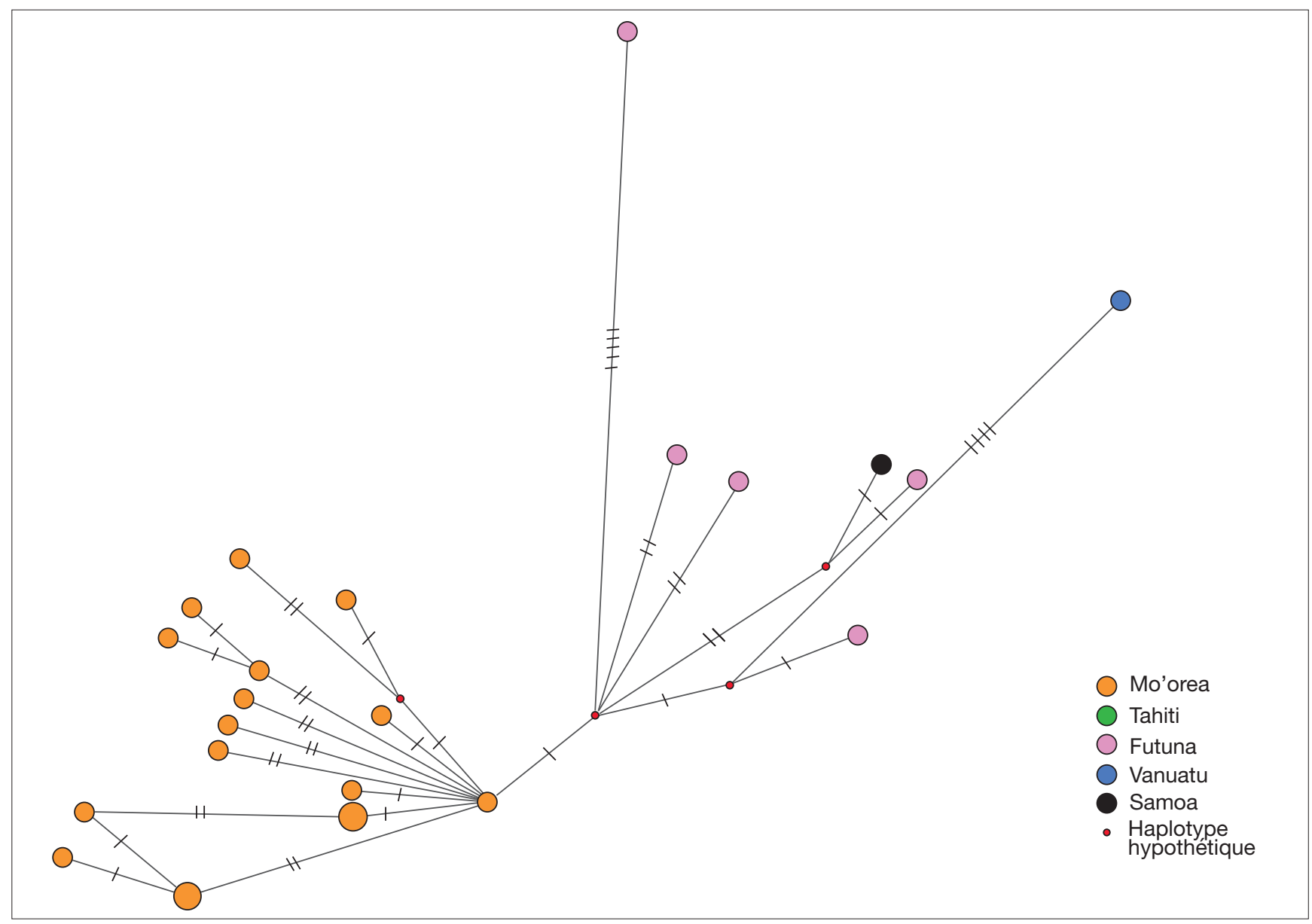

FIG. 5. - Réseau d'haplotypes de Neritina canalis Sowerby, 1825 selon la méthode du median-joining. Les cercles sont proportionnels à la fréquence des occurences, le nombre de mutations est indiqué sur les branches par des tirets.

haplogroupe est partagé par sept échantillons des Comores (six de Mohéli, un de Anjouan) et un autre est à la fois aux Comores et aux Seychelles.
S'agissant de $N$. canalis, les $609 \mathrm{pb}$ du gène COI séquencées pour 24 individus, ont donné 22 haplotypes, 38 (6\%) sites polymorphes, une diversité haplotypique très élevée 
TABLEAU 2. - Indices de diversité basés sur 609 pb du gène COI chez les deux populations de Neritina stumpffi Boettger, 1890. Abréviations et symboles : ns, nombre de séquences; $\mathbf{n h}$, nombre d'haplotypes; $\mathbf{n s p}$, nombre de sites polymorphes; $\mathbf{h}$, diversité haplotypique; $\boldsymbol{\pi}$, diversité nucléotidique.

\begin{tabular}{lrrrrc}
\hline & ns & nh & nsp & h & $\mathbf{\pi}$ \\
\hline océan Indien & 28 & 19 & 41 & 0,937 & 0,015 \\
océan Pacifique & 6 & 6 & 15 & 1,000 & 0,009 \\
\hline
\end{tabular}

TABlEAu 3. - Indices de diversité basés sur 609 pb du gène COI chez les deux populations de Neritina canalis Sowerby, 1825. Abréviations et symboles ns, nombre de séquences; nh, nombre d'haplotypes; nsp, nombre de sites polymorphes; $\mathbf{h}$, diversité haplotypique; $\boldsymbol{\pi}$, diversité nucléotidique.

\begin{tabular}{lrrrrc}
\hline & ns & nh & nsp & h & $\boldsymbol{\pi}$ \\
\hline Pacifique ouest & 7 & 7 & 19 & 1,000 & 0,010 \\
Pacifique central & 17 & 15 & 19 & 0,985 & 0,006 \\
\hline
\end{tabular}

( $h=1,00$ dans le Pacifique ouest $\mathrm{h}=0,99$ dans le Pacifique central) et une diversité nucléotidique faible, plus basse en Pacifique central qu'en Pacifique ouest (voir Tableau 3). Le $F_{S T}$ estimé $(0,248)$ indique une forte structuration génétique entre Pacifique ouest et Pacifique central $(p=0,000)$. Ce résultat est soutenu par le réseau d'haplotypes (Fig. 5) qui montre deux principales entités génétiques, géographiquement distribuées entre Pacifique ouest (représenté par Samoa, Vanuatu et Futuna) et Pacifique central (représenté par Mo'orea et Tahiti). Les 17 échantillons du Pacifique central sont représentés par 14 haplotypes enracinés sur un même quinzième, et les sept du Pacifique ouest sont représentés par sept haplotypes différents.

\section{DISCUSSION}

Dans le cas des deux espèces étudiées, nous avons observé une structure génétique des populations et des variations de connectivité. La diversité génétique de toutes les populations observées dans cette étude est caractérisée par une diversité haplotypique élevée combinée à une diversité nucléotidique faible. Cela suggérerait une croissance rapide à partir d'une population ancestrale de faible effectif, si l'on pouvait écarter un effet artefactuel éventuel, dû au petit nombre d'échantillons issus du Pacifique ouest pour $N$. canalis, et de l'océan Pacifique pour $N$. stumpffi. S'agissant de $N$. stumpffi, les analyses génétique et morphométrique réalisées par Abdou et al. (2017) ont écarté l'hypothèse de deux entités génétiques vivant en sympatrie dans l'océan Indien. Par conséquent, la mise en évidence d'une structuration génétique chez cette espèce $\left(\mathrm{F}_{\mathrm{ST}}\right.$ $=0,288 ; 11$ mutations nucléotidiques) semble indiquer la présence d'une barrière (actuelle ou passée), qui limiterait la dispersion de l'espèce entre les deux océans, Pacifique et Indien. Néanmoins, des échantillons des Comores et des Seychelles, issus des mêmes stations possèdent des haplotypes différents, dont certains sont partagés avec des îles du Pacifique. Des migrations ont donc eu lieu du Pacifique vers l'océan Indien, même si elles ont été limitées.
De nombreuses études ont montré que, suivant des processus complexes de dispersion, des espèces aquatiques indopacifiques ont pu former ou non des populations distinctes, chez des gastéropodes (Frey \& Vermeij 2008 ; Crandall et al. 2008, 2010; Postaire et al. 2014 ; cette étude), des poissons (Gaither et al. 2010; Lord et al. 2012; Taillebois et al. 2013) ou des crustacés (Castelin et al. 2013). Avec ses milliers d'îles, l'AIA, également appelé Triangle de corail, est une des régions du monde les plus riches en biodiversité, dans laquelle les changements eustatiques induits par les glaciations du Pléistocène ont joué comme des pompes à spéciation par vicariance (Frey \& Vermeij 2008; Carpenter et al. 2011; de Bruyn et al. 2012). Mais si cette région constitue un haut lieu de la biodiversité actuelle, c'est essentiellement en raison de l'éclatement de la Téthys à l'oligocène/miocène, qui a provoqué une redistribution de taxons, y compris de la faune d'eau douce (Bunje \& Lindberg 2007). En effet, le centre de la paléobiodiversité marine tropicale se situait au sein de la Téthys (Cowman \& Bellwood 2013; Leprieur et al. 2016) jusqu'à sa migration vers le Triangle de corail au miocène, à la faveur de la fermeture de cet océan et à la délocalisation du bloc australien vers l'Asie du sud (Cowman \& Bellwood 2013; Postaire et al. 2014; Leprieur et al. 2016).

L'hypothèse de cette migration dans le sens océan Pacifique vers l'océan Indien est soutenue, non seulement par le réseau d'haplotypes et les indices de diversité génétique, mais aussi par la courantologie (Fig. 6). En effet, la diversité haplotypique maximale $(\mathrm{h}=1,00)$ dans l'océan Pacifique est en faveur d'une origine Pacifique de cette espèce.

À ce jour, $N$. stumpffi n'est signalée, dans l'océan Indien, qu'à Madagascar, aux Comores et aux Seychelles (Starmühlner 1983). Son absence aux Mascareignes (Griffiths \& Florens 2006) pourrait s'expliquer par la circulation des courants océaniques (Fig. 6) et/ou un comportement particulier de l'espèce (migration verticale, réponses sensorielles, etc.), le transport des larves vers l'habitat d'implantation approprié pouvant être influencé par l'interaction de facteurs biophysiques et comportementaux, notamment (Weersing \& Toonen 2009). Ces courants océaniques expliqueraient aussi le sens de migration, même si elle est limitée, entre l'océan Pacifique et l'océan Indien.

En effet, la connexion marine entre l'océan Pacifique et le sud-ouest de l'océan Indien (SOOI) est essentiellement assurée par le courant sud-équatorial (CSE), qui passe par un couloir délimité par le nord de l'Australie et le sud de l'Indonésie, entre $10^{\circ} \mathrm{S}$ et $20^{\circ} \mathrm{S}$. La circulation du CSE dans le SOOI (Fig. 6) a été expliquée par Guyomard et al. (2006) et modélisée en 3D par Pous et al. (2010), qui ont reproduit les voies possibles de migration des larves, en tenant compte notamment des courants, de la température et de la salinité. Il apparaît que ce courant, qui est le plus intense à la surface mais atteint $1400 \mathrm{~m}$ de profondeur par endroits (Pous et al. 2014), se divise en deux au niveau de Madagascar, après avoir traversé le plateau des Mascareignes, entre Carcados Carajos et l'île Maurice, pour donner naissance au courant sud-est malgache (CSEM) vers le sud, rejoignant le système du courant des Aiguilles, et au courant nord-est malgache (CNEM) (Fig. 6). Les simulations 


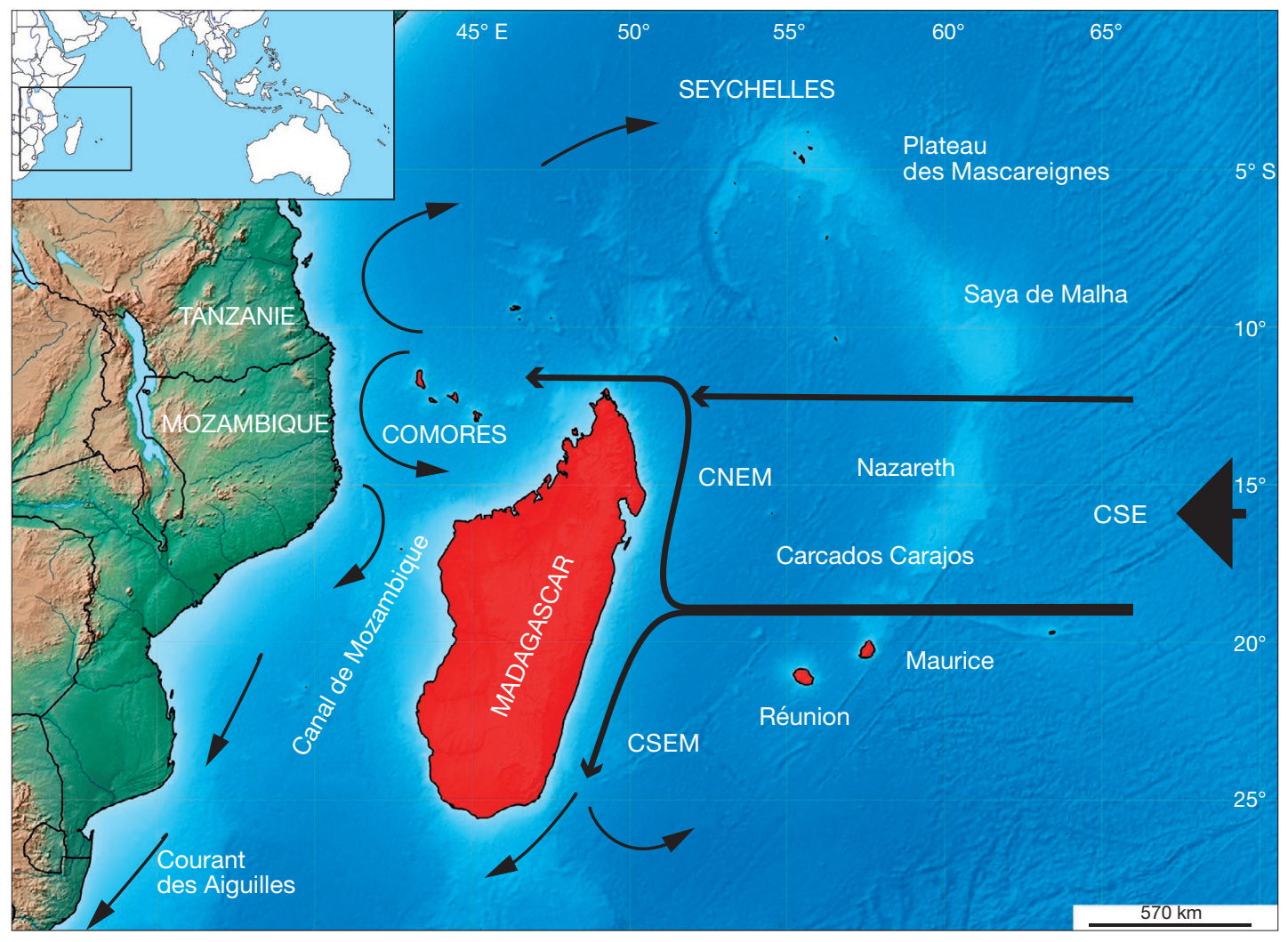

FIG. 6. - Circulation des courants océaniques dans le Sud ouest de l'océan Indien. Schéma basé sur les études de Guyomard et al. (2006) et Pous et al. (2010).

réalisées ont montré que l'essentiel des particules atteignant les Comores sont transportées par le flux nord du CSE, passant entre le banc de Saya de Malha et le banc de Nazareth, avant de rejoindre le CNEM au niveau du cap d'Ambre au nord de Madagascar (Pous et al. 2010, 2014). Le courant nord continue ensuite vers la côte est-africaine, au niveau de l'archipel de Quirimbas au nord du Mozambique, où il se subdivise en deux branches, l'une alimentant le courant côtier est-africain (Guyomard et al. 2006) vers le Nord, l'autre, entrant dans la circulation anticyclonique du nord du canal (Fig. 6). Cette voie de migration directe, par le nord du CSE entre $10^{\circ} \mathrm{S}$ et $14^{\circ} \mathrm{S}$, serait plus courte et sans doute plus rapide pour les larves des espèces diadromes, les courants y étant plus intenses. C'est ce que suggèrent Castelin et al. (2013), dans leur étude sur la phylogéographie de la crevette amphidrome Macrobrachium lar (Fabricius, 1798), qui trouvent des résultats similaires aux nôtres. Ils expliquent ainsi l'existence d'haplotypes partagés entre des échantillons du Pacifique ouest (Indonésie, Îles Caroline, Nouvelle-Calédonie, Vanuatu, Fidji et Futuna) et des échantillons du nord du SOOI (Comores et Seychelles), alors qu'ils observent au sein même de cette zone une forte rupture phylogénétique entre les échantillons de Madagascar, Maurice et La Réunion, d'une part et ceux des Comores et des Seychelles d'autre part. Lord et al. (2012) ont également trouvé pour le téléostéen amphidrome Sicyopterus lagocephalus (Pallas, 1770), une forte structuration géographique entre la Polynésie française et le Pacifique ouest, de même qu'entre le Pacifique ouest et l'océan Indien.
Selon les résultats de ces études et le schéma observé pour $N$. stumpffi, on peut raisonnablement émettre l'hypothèse que les larves pourraient emprunter du Pacifique, la route migratoire du nord de l'océan Indien, passant entre Saya de Malha et Nazareth, puisque nous avons trouvé aux Comores et aux Seychelles des haplotypes du Pacifique. Cette hypothèse est renforcée par l'absence de cette espèce dans les Mascareignes (Griffiths \& Florens 2006), et par le fait que sa présence à Madagascar est essentiellement signalée à Nossy Bé et Nossy Komba (Boettger 1890; Starmühlner 1983), au nord-ouest de l'île, à hauteur des Comores. Notons cependant que si le rôle majeur des courants océaniques dans la structuration génétique des populations est incontestable (White et al. 2010; Pages et al. 2013), il existe plusieurs cas montrant qu'à une plus grande échelle géographique, des espèces, parfois proches, subissant les mêmes courants océaniques, empruntent des routes de migration différentes, puisque les traits d'histoire de vie particuliers des espèces (la profondeur à laquelle elles circulent, par exemple) interviennent. Le cas des gastéropodes amphidromes Neritina canalis et Neripteron dilatatum (Broderip, 1833) (Crandall et al. 2010), des gastéropodes marins Nerita plicata (Linnaeus, 1758) et $N$. albicilla (Linnaeus, 1758) (Crandall et al. 2008) ou celui des poissons téléostéens récifaux Lutjanus kasmira (Forsskål, 1775) et L. fulvus (Forster, 1801) (Gaither et al. 2010), illustrent bien ce phénomène.

La forte structuration génétique de $N$. canalis $\left(F_{S T}=0,248\right)$ que nous avons observée entre le Pacifique ouest et le Pacifique central, est en accord avec les résultats obtenus dans l'étude réalisée 
par Crandall et al. (2010) sur cette même espèce. Cependant, nous n'avons trouvé aucun haplotype partagé entre le Pacifique ouest et le Pacifique central, cela étant probablement dû au faible nombre d'individus analysés $(\mathrm{N}=24)$. Il existerait donc une barrière à la dispersion entre ces deux zones. L'existence de cette barrière entre le Pacifique central, la Polynésie en particulier, et le Pacifique ouest, a été également trouvée pour deux espèces amphidromes à très large répartition indo-pacifique, l'une d'un poisson gobie (Sicyopterus lagocephalus) (Keith et al. 2005; Lord et al. 2012) et l'autre d'une crevette (Macrobrachium lar) (Castelin et al. 2013). Cet isolement serait dû à une combinaison complexe d'obstacles géographique, biogéographique ou écologique. S'agissant de N. canalis, Crandall et al. (2010) ont, en outre, montré l'absence de structure géographique au sein et entre trois archipels du Pacifique ouest (Vanuatu, Fidji et Samoa), sur une étendue de plus de $2000 \mathrm{~km}$, prouvant ainsi la grande capacité de cette espèce à retarder la métamorphose. Dans l'étude de Crandall et al. (2010), une autre espèce de nérite, Neripteron dilatatum, ne montre aucune structure génétique à l'échelle de plusieurs milliers de kilomètres, allant du Pacifique ouest aux Îles de la Société et aux Marquises, montrant aussi la perméabilité de la barrière entre le Pacifique ouest et le Pacifique central dans le cas de cette espèce. Cela peut s'expliquer par des traits de vie différents, par exemple par une très longue durée de vie larvaire ou par un phénomène d'extinction suivi de recolonisation. Selon Crandall et al. (2012), la connexion entre le Pacifique central et le Pacifique ouest dans ce cas, et donc le flux génétique entre ces deux biozones, se ferait essentiellement lors des variations des courants océaniques, dues à des événements climatiques de grande ampleur, El Niño et La Niña, ayant cours périodiquement dans cette région, et connus sous le nom de "El Niño Southern Oscillation».

Il apparait clairement que l'étude de la dispersion et de la connectivité des espèces est essentielle à la gestion durable des ressources naturelles aquatiques marines et dulçaquicoles, et que les protocoles de gestion doivent nécessairement tenir compte des voies de dispersion (Wilson et al. 2016). Maintenant que la répartition géographique des deux espèces de nérites étudiées ici est relativement mieux connue, il est souhaitable que, dans une perspective de gestion et de conservation, des études plus approfondies soient réalisées, surtout pour $N$. stumpffi, à des échelles locales et/ou régionales. L'anthropisation croissante des milieux aquatiques insulaires pouvant impacter négativement le cycle vital de ces espèces, une connaissance fine de leurs habitats aidera à les préserver, sachant bien évidemment que la condition principale reste le maintien d'un corridor écologique continu et permanent entre l'océan et les habitats dulçaquicoles (Keith 2003; Abdou et al. 2015).

\section{CONCLUSION}

Notre étude soutient l'existence de deux barrières biogéographiques partielles régulant la circulation des larves de nérites au sein de l'Indo-Pacifique au cours du temps. La première entre le Pacifique ouest et le Pacifique central, ce que d'autres études avaient déjà signalé chez des gastéropodes (Crandall et al. 2010, 2012), des crustacés (Castelin et al. 2013) ou des poissons amphidromes (Lord et al. 2012). La deuxième entre l'océan Indien et le Pacifique, également signalé pour d'autres groupes.

Notre étude soutient également le fait que la barrière indopacifique a été plus ou moins perméable selon les espèces (Carpenter et al. 2011). Ce qui est le cas de l'une des deux espèces étudiées ici, $N$. stumpffi. La phylogéographie de cette espèce nécessite des études complémentaires, avec une augmentation de l'échantillonnage aussi bien pour le nombre des échantillons que pour celui des localités échantillonnées, dans le Pacifique comme dans l'océan Indien. Il serait aussi intéressant de pouvoir dater les événements et d'évaluer l'importance des flux géniques.

\section{Remerciements}

Nous remercions D. Labrousse, G. Marquet et L. Taillebois, qui ont récolté des échantillons, ainsi que l'UMR 7208 BOREA et l'USR 3278 CRIOBE qui ont financé la mission de terrain du premier auteur à Mo'orea en 2014. Nous tenons à remercier également les rapporteurs, dont les recommandations nous ont permis d'améliorer cet article.

\section{RÉFÉRENCES}

AbDou A. \& Bouchet P. 2000. — Nouveaux gastéropodes Endodontidae et Punctidae (Mollusca : Pulmonata) récemment éteints de l'archipel des Gambier (Polynésie). Zoosystema 22 (4): 689-707.

Abdou A., Muratov I. \& Bouchet P. 2008. - Streptaxid microcosm: the radiation of the endemic genus Pseudelma on Mayotte, Comores Islands (Gastropoda, Pulmonata, Streptaxidae). Tropical Zoology 21 (1): 123-151.

AbDou A., Keith P. \& GAlzin R. 2015. - Freshwater neritids (Mollusca: Gastropoda) of tropical islands: amphidromy as a life cycle, a review. Revue d'Écologie (Terre et Vie) 70 (4): 387-397.

Abdou A., Galzin R., Lord C., Denys G. \& Keith P. 2017. Revision of the species complex 'Neritina pulligera' (Gastropoda: Cycloneritimorpha: Neritidae) using taxonomy and barcoding. Vie et Milieu - Life \& Environment 67: (3-4).

Alda F., Gagne R. B., Walter R. P., Hogan J. D., Moody K., Zink F., McIntyre P. B., Gilliam J. F. \& Blum M. J. 2016. Colonization and demographic expansion of amphidromous stream fauna across the Hawaiian Archipelago. Journal of Evolutionary Biology 29 (10): 2054-2069. https://doi.org/10.1111/jeb.12929

AVISE J. C. 1998. - The history and purview of phylogeography: A personal reflection. Molecular Ecology 7: 371-379. https://doi. org/10.1046/j.1365-294x.1998.00391.x

Avise J. C., J. Arnold R. M. Ball E. Bermingham T. Lamb J. E. Neigel C. A. Reeb \& Saunders N. C. 1987. - Intraspecific Phylogeography: The Mitochondrial DNA Bridge Between Population Genetics and Systematics. Annual Review of Ecology and Systematics 18: 489-522. https://doi.org/10.1146/annurev. es.18.110187.002421

Ayre D. J., Minchinton T. E. \& Perrin C. 2009. — Does life history predict past and current connectivity for rocky intertidal invertebrates across a marine biogeographic barrier? Molecular Ecology 18 (9): 1887-1903. https://doi.org/10.1111/j.1365-294X.2009.04127.x

BANDELT H.-J., FORSTER P. \& RÖHL A. 1999. — Median-joining networks for inferring intraspecific phylogenies. Molecular Biology and Evolution 16: 37-48. https://doi.org/10.1093/oxfordjournals. molbev.a026036 
BoetTGer 1890. — Zur Kenntnis der Land- und Süßwassermollusken von Nossi-Bé. II. Nachrichtsblatt der Malakozoologischen Gesellschaft 22: 81-137.

Bunje P. M. E. \& LindBERG D. R. 2007. — Lineage divergence of a freshwater snail clade associated with post-Tethys marine basin development. Molecular Phylogenetics and Evolution 42 (2): 373387. https://doi.org/10.1016/j.ympev.2006.06.026

Carpenter K. E., Barber P. H., Crandall E. D., Ablan-Lagman Ma. C. A., Ambariyanto, Mahardika G. N., Manjaji-Matsumoto B. M., Juinio-MeŇEz M. A., Santos M. D., Starger C. J. \& TOHA A. H. A 2011. — Comparative Phylogeography of the Coral Triangle and Implications for Marine Management. Journal of Marine Biology 2011. https://doi.org/10.1155/2011/396982

CASTELIN M. 2010. — Lien entre endémisme et développement larvaire en milieu marin. Le cas des gastéropodes des monts sous-marins de la Zone Economique Exclusive de Nouvelle Calédonie. Thèse de doctorat du Muséum national d'histoire naturelle, Paris, 275 p.

Castelin M., Feutry P., Hautecoeur M., Marquet G., Wowor D., ZimmermanN G., KeITH P. 2013. - New insight on the genetic connectivity of the widespread amphidromous prawn Macrobrachium lar (Fabricius, 1798) (Crustacea: Decapoda: Palaemonidae). Marine Biology 160: 1395-1406.

CovicH A. P. 2006. - Dispersal - limited biodiversity of tropical insular streams. Polish Journal of Ecology 54 (4): 523-547.

Cowie R. H., Evenhuis N. L. \& Christensen C. C. 1995. Catalog of the Native Land and Freshwater Molluscs of the Hawaiian Islands. Leiden: Backhuys Publishers, 248 p.

Cowman P. F. \& BellWoOD D. R. 2013. - The historical biogeography of coral reef fishes: global patterns of origination and dispersal. Journal of Biogeography 40: 209-224. https://doi. org/10.1111/jbi. 12003

Crandall E. D., Taffel J. R. \& Barber P. H. 2010. - High gene flow due to pelagic larval dispersal among South Pacific archipelagos in two amphidromous gastropods. Heredity 104: 563-572. https://doi.org/10.1038/hdy.2009.138

Crandall E. D., Treml E. A. \& Barber P. H. 2012. - Coalescent and biophysical models of stepping-stone gene flow in neritid snails. Molecular Ecology 21 (22): 5579-5598.

Crandall E. D., Frey M. A., Grosberg R. K. \& Barber P. H., 2008. - Contrasting demographic history and phylogeographical patterns in two Indo-Pacific gastropods. Molecular ecology 17 (2): 611-626. https://doi.org/10.1111/j.1365-294X.2007.03600.x

de Bruyn M., von Rintelen K., von Rintelen T., Mather P. B. \& CARVALHO G. R. 2012. - Molecular biogeography and phylogeography of the freshwater fauna of the Indo-Australian Archipelago, in GOWER D., JOHNSON K., RichardSON J., Rosen B., RÜBER L. \& Williams S. (eds), Biotic Evolution and Environmental Change in Southeast Asia, 316-347. [Online]. Systematics Association Special Volume Series. Cambridge: Cambridge University Press. Available from: Cambridge Books Online. https://doi.org/10.1017/CBO9780511735882.015 [Accessed 29 July 2015].

Excoffier L., Smouse P. E. \& QuatTro J. M. 1992. — Analyse of molecular variance inferred from metric distance among DNA haplotypes: application to human mitochondrial DNA restriction data. Genetics 131: 479-491.

EXCOFFIER L. \& LISCHER H. E. L. 2010. - Arlequin suite ver 3.5: A new series of programs to perform population genetics analyses under Linux and Windows. Molecular Ecology Resources 10: 564-567. https://doi.org/10.1111/j.1755-0998.2010.02847.x

Feutry P., Valade P., Ovenden J. R., Lopez P. J. \& Keith P. 2012. - Pelagic larval duration of two diadromous Kuhliidae species (Teleostei: Percoidei) from Indo-Pacific insular systems. Marine and Freshwater Research 63: 397-402. https://doi. org/10.1071/MF11243

Feutry P., Vergnes A., Broderick D., Lambourdière J., Keith P. \& Ovenden J. R. 2013. - Stretched to the limit. Can a shortpelagic larval duration connect adult populations of an Indo-
Pacific diadromousfish (Kublia rupestris)? Molecular Ecology 22: 1518-1530. https://doi.org/10.1111/mec. 12192

Folmer O., Black M., Hoeh W., Lutz R. \& Vrijenhoek R. 1994. - DNA primers for amplification of mitochondrial cytochrome oxidase subunit I from diverse metazoan invertebrates. Molecular Marine Biology and Biotechnology 3: 294-299.

Frey M. A. \& Vermeij G. J. 2008. — Molecular phylogenies and historical biogeography of a circumtropical group of gastropods (Genus: Nerita): Implications for regional diversity patterns in the marine tropics. Molecular Phylogenetics and Evolution 48: 10671086. https://doi.org/10.1016/j.ympev.2008.05.009

Gaither M. R., Toonen R. J., Robertson D. R., Planes S. \& BOWEN B. W. 2010. — Genetic evaluation of marine biogeographical barriers: perspectives from two widespread Indo-Pacific snappers (Lutjanus kashmira and Lutjanus fluvus). Journal of Biogeography 37: 133-147.

GARGOMINY O. \& FonTAINE B. 2014. - A global overview of the terrestrial and freshwater molluscs, in MEYER J. Y. \& ClARIDGE E. M. [Eds]. Terrestrial Biodiversity of the Austral Islands, French Polynesia. Muséum national d'Histoire naturelle, Paris, (Patrimoines naturels) 72: 55-91.

GERLACH J. 2006. - Terrestrial and Freshwater Mollusca of Seychelles. Leiden: Backhuys Publishers, $117 \mathrm{p}$.

Griffiths O. \& Florens V. 2006. - A Field Guide to the NonMarine Molluscs of the Mascarene Islands (Mauritius, Rodrigues and Réunion) and the Northern Dependencies of Mauritius. Bioculture Press, Mauritius, $185 \mathrm{p}$.

Guyomard D., Petit M., Desruisseaux M., Stretta J.-M. \& GARDEL L. 2006. - Hydroclimat du sud-ouest de l'océan Indien et océanographie spatiale, in PETIT M. (ed.) \& HuYNH F. (ed.). Halieutique et environnement océanique : le cas de la pêche palangrière à l'espadon depuis l'île de la Réunion. Paris, IRD: 39-65.

HANSEN T. A. 1980. - Influence of larval dispersal and geographic distribution on species longevity in neogastropods. Paleobiology 6 (2): 193-207. https://doi.org/10.1017/S0094837300025756

HAYNES A. 2001. - Freshwater snails of the tropical pacific islands. Institute of Applied Sciences, Suva, 116 p.

HAYNES A. 2005. - An evaluation of members of the genera Clithon Montfort, 1810 and Neritina Lamarck 1816 (Gastropoda: Neritidae). Molluscan Research 25 (2): 75-84.

HolTHUIS B. V. 1995. - Evolution between marine and freshwater habitats: a case study of Gastropod suborder Neritopsina. PhD thesis, University of Washington DC, USA, 116 p.

KANO Y. 2006. - Usefulness of the opercular nucleus for inferring early development in neritimorph gastropods. Journal of Morphology 267: 1120-1136. https://doi.org/10.1002/jmor.10458

KANO Y. 2009. - Hitchhiking behaviour in the obligatory upstream migration of amphidromous snails. Biology Letters 5: 465-468. https://doi.org/10.1098/rsbl.2009.0191

KANO Y. \& KASE T. 2003. - Systematics of the Neritilia rubida complex (Gastropoda: Neritiliidae): three amphidromous species with overlapping distributions in the Indo-Pacific. Journal of Molluscan Studies 69: 273-284. https://doi.org/10.1093/ mollus/69.3.273

KANO Y. \& KASE T. 2004. - Genetic exchange between anchialine cave populations by means of larval dispersal: the case of a new gastropod species Neritilia cavernicola. Zoologica Scripta 33: 423-437.

KANO Y. \& KASE T. 2008. - Diversity and distributions of the submarine-cave Neritiliidae in the Indo-Pacific (Gastropoda: Neritimorpha). Organisms Diversity \& Evolution 8: 22-43. https:// doi.org/10.1016/j.ode.2006.09.003

KeITH P. 2003. - Biology and ecology of amphidromous Gobiidae in the Indo-pacific and the Caribbean regions. Journal of Fish Biology 63 (4): 831-847. https://doi.org/10.1046/j.10958649.2003.00197.x

Keith P., Galewski T., Gattaneo-Berrebi G., Hoareau T. \& BerRebi P. 2005. - Ubiquity of Sicyopterus lagocephalus (Teleostei: Gobiodei) and phylogeography of the genus Sicyopterus in 
the Indo-Pacific area inferred from mitochondrial cytochrome $b$ gene. Molecular Phylogenetics and Evolution 37: 721-732. https:// doi.org/10.1016/j.ympev.2005.07.023

KeITH P., ABDou A. \& LABAT J.-N. 2006. — Inventaire faunistique des rivières des Comores et Inventaire botanique. Rapport. Muséum national d'Histoire naturelle, Paris, 109 p.

Leprieur F., Descombes F., Gaboriau T., Cowman P. F., Parravicin V., Kulbicki M., Melián C. J., de Santana C. N., Heine C., Mouillot D., Bellwood D. R. \& Pellissier L. 2016. — Plate tectonics drive tropical reef biodiversity dynamics. Nature Communications 7: 11461. https://doi.org/10.1038/ncomms11461

Librado P. \& Rozas J. 2009. - DnaSP v5: A software for comprehensive analysis of DNA polymorphism data. Bioinformatics, 25: 1451-1452. https://doi.org/10.1093/bioinformatics/btp187

LiU H. T. T. \& ResH V. H. 1997. - Abundance and microdistribution of freshwater gastropods in three streams of Moorea, French Polynesia. Annales de Limnologie 33: 235-244.

Lord C., Brun C., Hauteceur M. \& KeITH P. 2010. — Insights on endemism: comparison of the duration of the marine larval phase estimated by otolith microstructural analysis of three amphidromous Sicyopterus species (Gobiidae: Sicydiinae) from Vanuatu and New Caledonia. Ecology of Freshwater Fish 19: 26-38.

Lord C., Lorion J., Dettai A., Watanabe S., Tsukamoto K., Cruaud C. \& Keith P. 2012. - From endemism to widespread distribution: phylogeography of three amphidromous Sicyopterus species (Teleostei: Gobioidei: Sicydiinae). Marine Ecology Progress Series 455: 269-285. https://doi.org/10.3354/meps09617

MCDOWALl R. M. 1992. - Diadromy: origins and definition of terminology. Copeia 1: 248-251. https://doi.org/10.2307/1446563

MCDOWALl R. M. 2007. — On amphidromy, a distinct form of diadromy in aquatic organisms. Fish and Fisheries 8: 1-13. https:// doi.org/10.1111/j.1467-2979.2007.00232.x

MCDOWALL R. M. 2010. - Why be amphidromous: expatrial dispersal and the place of source and sink population dynamics? Reviews in Fish Biology and Fisheries 20: 87-100. https://doi. org/10.1007/s11160-009-9125-2

Muratov I., Abdou A. \& Bouchet P. 2005. - Charopid land snails (Gastropoda Pulmonata Charopidae) from Mayotte, Comores: alive and well. Tropical Zoology 18: 171-208. https:// doi.org/10.1080/03946975.2005.10531220

Myers M. J., Meyer C. P. \& Resh V. H. 2000. - Neritid and thiarid gastropods from French Polynesian streams: how reproduction (sexual, parthenogenetic) and dispersal (active/passive) affect population structure. Freshwater Biology 44: 535-545.

Page T. J., Torati L. S., Cook B. D., Binderup A., Pringle C. M., Reuschel S., Schubart CH. D. \& Hughes J. M. 2013. Invertébrés Sans Frontières: Large Scales of Connectivity of Selected Freshwater Species among Caribbean Islands. Biotropica 45 (2): 236-244. (Première mise en ligne : 3.X.2012) https:// doi.org/10.1111/j.1744-7429.2012.00900.x

Pointier J. P. \& MARQUeT G. 1990. - Taxonomy and distribution of freshwater mollusks of French Polynesia. Venus 49 (3): 215-231.

Postaire B., Bruggemann J. H., Magalon H. \& Faure B. 2014. - Evolutionary Dynamics in the Southwest Indian Ocean Marine Biodiversity Hotspot: A Perspective from the Rocky Shore Gastropod Genus Nerita. PLoS ONE 9(4): e95040. https://doi. org/10.1371/journal.pone.0095040

Pous S., Feunteun E. \& Ellien C. 2010. — Investigation of tropical eel spawning area in the South-Western Indian Ocean: Influence of the oceanic circulation. Progress in Oceanography 86 (3): 396-413. https://doi.org/10.1016/j.pocean.2010.06.002

Pous S., lazure P., André G., Dumas F., Halo I. \& Penven P. 2014. - Circulation around La Réunion and Mauritius islands in the south-western Indian Ocean: A modeling perspective. Journal of Geophysical Research: Oceans 119: 1957-1976. https:// doi.org/10.1002/2013JC009704

Resh V. H., Barnes J. R. \& Craig D. A. 1990. - Distribution and ecology of benthic invertebrates in the Opunohu river catchment, Moorea, French Polynesia. Annales de Limnologie 26: 195-214.

Resh V. H., Barnes J. R., Benis-STeger B. \& Craig D. A. 1992. Life-history features of some invertebrates in a French Polynesian stream. Studies on Neotropical Fauna and Environment 27:145-153.

Rijsdijk K. F., Hengl T., Norder S. J., Ávila S. P., \& FernándeZPALÁCIOS J. M. 2014. - Modelling sea level driven change of Macaronesian archipelago configurations since $120 \mathrm{kyr}$ BP., in Fernández-Palacios J.-M., de Nascimento L., HernánDez J. C., Clemente C., González A. \& Díaz-González J. P. (eds). Climate Change perspectives from the Atlantic: past, present and future. Servicio de Publicaciones, Universidad de La Laguna: 127-148.

STARMÜHLNER F. 1983. - Results of the hydrobiological mission 1974 of the zoological institute of the university of Vienna. Part VIII: Contributions to the knowledge of the freshwatergastropods of the Indian Ocean islands (Seychelles, Comores, Mascarene-Archipelagos). Annalen des Naturhistorischen Museums in Wien 84B: 127-249.

Taillebois L., Castelin M., Ovenden J. R., Bonillo C., Keith P. 2013. - Contrasting Genetic Structure among Populations of Two Amphidromous Fish Species (Sicydiinae) in the Central West Pacific. PLoS ONE, 8(10): e75465. https://doi.org/10.1371/ journal.pone. 0075465

Tamura K., Stecher G., Peterson D., Filipski A. \& Kumar S. 2013. - MEGA6: Molecular Evolutionary Genetics Analysis Version 6.0. Molecular Biology and Evolution 30 (12): 27252729. https://doi.org/10.1093/molbev/mst197

Valade P., Mary N., ABdou A. \& Keith P. 2007. - Inventaire des espèces de poissons et d'invertébrés des eaux douces de Mayotte. ARDA - Muséum national d'Histoire naturelle / DAF de Mayotte. Rapport final, August 2007, 144 p.

VANCE R. R. 1973. - On reproductive strategies in marine benthic invertebrates. The American Naturalist 107: 339-352.

VORIS H. K. 2000. - Maps of Pleistocene sea levels in Southeast Asia: shorelines, river systems and time durations. Journal of Biogeography 27 (5): 1153-1167. https://doi.org/10.1046/j.13652699.2000.00489.x

Waters J. M., King T. M., O'Loughlin P. M. \& Spencer H. G. 2005. - Phylogeographical disjunction in abundant high-dispersal littoral gastropods. Molecular Ecology 14 (9): 2789-2802. https://doi.org/10.1111/j.1365-294X.2005.02635.x

WeERSING K. \&TOONEN R. J. 2009. — Population genetics, larval dispersal, and connectivity in marine systems. Marine Ecology Progress Series 393: 1-12. https://doi.org/10.3354/meps08287

White C., Selkoe K. A., Watson J., Siegel D. A., Zacherl D. C. \& TOONEN R. J. 2010. - Ocean currents help explain population genetic structure. Proceedings of the Royal Society $B$ 277: 1685-1694.

Wilson L. J., Fulton C. J., Hogg A. M., Joyce K. E., Radford B. \& FRASER C. I. 2016. - Climate-driven changes to ocean circulation and their inferred impacts on marine dispersal patterns. Global Ecology and Biogeography 25: 923-939. https://doi. org/10.1111/geb.12456

WRIGHT S. 1951. - The genetical structure of populations. Annals of Eugenics 15:323-354. https://doi.org/10.1111/j.1469-1809.1949. tb02451.x

WRIGHT S. 1978. - Evolution and the genetics of populations. Variability within and among natural populations. Chicago, IL, USA: University of Chicago Press, 590 p. 